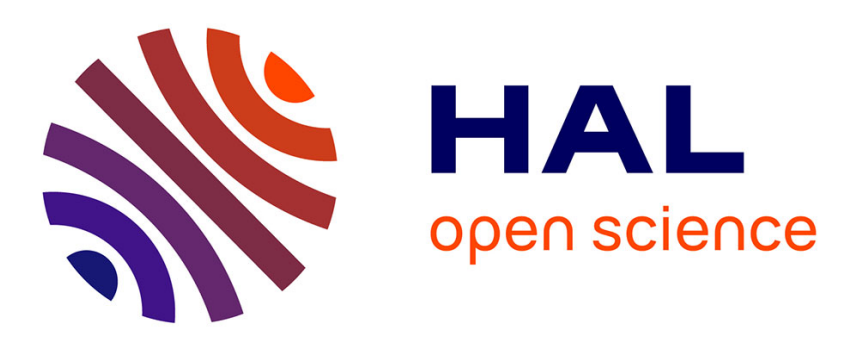

\title{
Coalescence of contaminated water drops at an oil/water interface: Influence of micro-particles
}

\author{
Erik de Malmazet, Frédéric Risso, Olivier Masbernat, Vincent Pauchard
}

\section{To cite this version:}

Erik de Malmazet, Frédéric Risso, Olivier Masbernat, Vincent Pauchard. Coalescence of contaminated water drops at an oil/water interface: Influence of micro-particles. Colloids and Surfaces A: Physicochemical and Engineering Aspects, 2015, vol. 482, pp. 514-528. 10.1016/j.colsurfa.2015.06.044 . hal-01332242

\section{HAL Id: hal-01332242 \\ https://hal.science/hal-01332242}

Submitted on 15 Jun 2016

HAL is a multi-disciplinary open access archive for the deposit and dissemination of scientific research documents, whether they are published or not. The documents may come from teaching and research institutions in France or abroad, or from public or private research centers.
L'archive ouverte pluridisciplinaire HAL, est destinée au dépôt et à la diffusion de documents scientifiques de niveau recherche, publiés ou non, émanant des établissements d'enseignement et de recherche français ou étrangers, des laboratoires publics ou privés. 


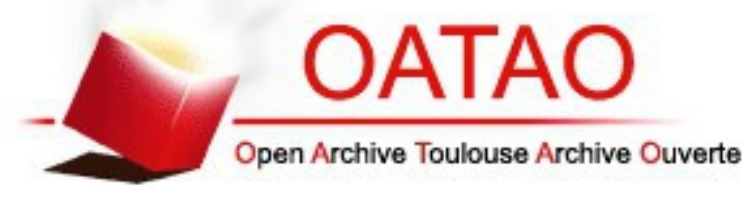

\section{Open Archive TOULOUSE Archive Ouverte (OATAO)}

OATAO is an open access repository that collects the work of Toulouse researchers and makes it freely available over the web where possible.

This is an author-deposited version published in : http://oatao.univ-toulouse.fr/ Eprints ID : 15869

To link to this article : DOI:10.1016/j.colsurfa.2015.06.044

URL : http://dx.doi.org/10.1016/j.colsurfa.2015.06.044

To cite this version : Malmazet, Erik de and Risso, Frédéric and Masbernat, Olivier and Pauchard, Vincent Coalescence of contaminated water drops at an oil/water interface: Influence of micro-particles. (2015) Colloids and Surfaces A: Physicochemical and Engineering Aspects, vol. 482. pp. 514-528. ISSN 0927-7757

Any correspondence concerning this service should be sent to the repository administrator: staff-oatao@listes-diff.inp-toulouse.fr 


\title{
Coalescence of contaminated water drops at an oil/water interface: Influence of micro-particles
}

\author{
Erik de Malmazet $^{\mathrm{a}, \mathrm{c}}$, Frédéric Risso ${ }^{\mathrm{a}, \mathrm{c}}$, Olivier Masbernat ${ }^{\mathrm{b}, \mathrm{c}, *}$, Vincent Pauchard ${ }^{\mathrm{d}, \mathrm{e}}$ \\ a Institut de Mécanique des Fluides, CNRS E Université de Toulouse, France \\ b Université de Toulouse INPT-UPS, Laboratoire de Génie Chimique, Toulouse, France \\ ' CNRS, Fédération de Recherche FERMaT, Toulouse, France \\ ${ }^{\mathrm{d}}$ Department of Process Technology, SINTEF Materials and Chemistry, Trondheim, Norway \\ e FACE, The Flow Assurance Center of Excellence, Norway
}

\section{H I G H L I G H T S}

- The role of micro-particles on dropinterface coalescence dynamics is investigated.

- Without particles drop lifetimes are randomly scattered between a 10 and $10^{4} \mathrm{~s}$.

- With particles, drop lifetime is reduced and correlated to drop diameter.

- As interface is aging, the lower the surface tension, the shorter the drop lifetime.

- Film retraction speed is controlled by interfacial tension, not by film thickness.

Keywords:

Coalescence time

Interfacial tension

Bridging effect

Film retraction speed
G R A P H I C A L A B S T R A C T

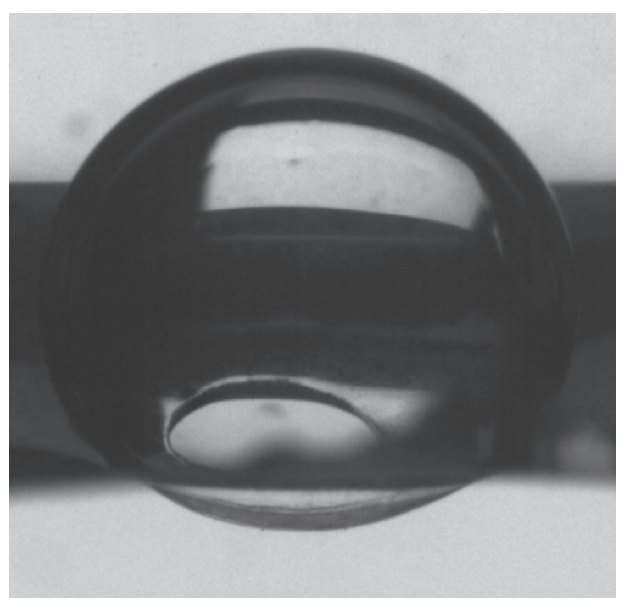

A B S T R A C T

The effect of micro-particles and interface aging on coalescence of millimetre-sized water drops with an oil/water interface is studied over long times. The system is not pure and interface contamination grows with time, resulting in a slow but continuous decrease of interfacial tension over time (from 35 to $10 \mathrm{mN} / \mathrm{m}$ ), which is measured in situ using an original technique. Without added micro-particles, coalescence times are randomly distributed and uncorrelated to drop diameter or interfacial tension. In presence of $10 \mu \mathrm{m}$ size hollow glass particles at the oil/water interface, coalescence times become more reproducible and show a clear dependence upon drop diameter and interface aging. Results are consistent with a classical drainage model assuming that the critical thickness at which interstitial film ruptures scales as the micro-particle diameter, a result that tends to validate the bridging scenario. Interestingly, the film retraction speed during the coalescence process does not follow theoretical predictions in a planar geometry. High-speed imaging of the retracting film reveals that the hole rim is bending upward while retracting, resulting in a strong slowdown of retraction speed. This is caused by the difference of interfacial tension between oil/drop freshly formed interfaces and the aged oil/water interface.

\footnotetext{
* Corresponding author.

E-mail address: olivier.masbernat@ensiacet.fr (O. Masbernat).
} 


\section{Introduction}

Understanding the physics of coalescence of drops and bubbles is an important issue for every industrial process where it is necessary either to separate the components of a dispersion, or to render this dispersion as stable as possible. In the oil industry for example, the produced crude oil is initially always mixed with some amount of water in the form of an emulsion, which must be processed to remove the dispersed water in order to meet crude oil specifications for transportation and storage. This water-oil separation process is usually based on gravitational separation, where the denser water droplets settle, aggregate, and ultimately coalesce. However, the kinetics of this process are quite complex because the coalescence is strongly influenced by temperature and by the presence of several adsorbed species and solid particles within the crude oil, and thus additional thermal, mechanical and chemical methods are usually used to enhance the gravitational separation process [1]. In this paper, we investigate experimentally an elementary coalescence mechanism occurring during this separation process: a water drop falling through a lighter oil phase and then arriving at a plane oil/water interface where it will rest and ultimately coalesce. The goal of our study is to better understand the influence on coalescence (coalescence times, retraction speeds) of interfacial contamination by both surfactants and micro-particles.

The drop/interface coalescence phenomenon has been extensively studied in the past in the case of pure fluids or with added surfactants. One important result obtained from early studies [2-6] is that coalescence times are not reproducible despite the great care taken in all these works in limiting contamination by surfactants and micro-particles. Therefore, it was considered that the coalescence mechanism was probabilistic in nature, and that it is better described by statistics. Thus, most results on coalescence times are presented until now in the form of time distributions from which it is possible to define mean, median, minimum and maximum coalescence times. These distributions were found to be quite reproducible, and some clear influence of drop diameter and temperature were observed: in most cases, the mean and medium coalescence times increased with drop diameter and system temperature.

However, theoretical models developed to describe the probabilistic nature of coalescence, based on mechanical and thermal instabilities occurring during the film drainage, were in general not very convincing in predicting the observed time distributions. The other important result observed from these early studies is the very strong influence of surfactants over the coalescence process: by preventing interfacial mobility due to the Marangoni effect, the film drainage is decreased considerably, and thus the coalescence times are greatly increased.

We can then mention the works of Hartland [7-10], Hodgson and Lee [11], Jones and Wilson [12] and Basheva et al. [13]. In Hartland [7-9], although there are no results on coalescence times, other aspects of drop/interface coalescence have been studied in detail such as the drop shape when resting on a plane interface, the variation of film thickness with time and radial position and the film retraction. Hodgson and Lee [11] is a quite significant experimental work because contrary to most of other studies (past or future), almost perfectly reproducible coalescence times were obtained in some conditions, probably due to a special interface cleaning device that was used. This somehow contradicts the probabilistic description of the coalescence process. Jones and Wilson [12] is an important theoretical work on the film drainage problem of a drop over a plane interface, which shows the importance of interface mobility and the narrowing of the film at its periphery (the so-called dimple). Finally, the work of Basheva et al. [13] showed experimentally for the first time the dependence of the coalescence time on drop diameter in a wide range where two distinct coalescence regimes have been predicted: a Taylor regime for very small drops where there is no drop deformation and the film lifetime decreases with drop diameter (lubrication regime), and a Reynolds regime for large drops where there is drop deformation and the film lifetime increases with drop diameter (independently of the interface mobility). As a result, the coalescence time as a function of drop diameter passes through a minimum.

Many other works can also be mentioned, such as Hogdson and Woods [14], Lang and Wilke [15,16], Woods and Burrill [17], and more recently, Mohamed-Kassim and Longmire [18], Chen et al. [19] and Bozzano and Dente [20]. However, despite the numerous theoretical and experimental works on coalescence, it is still until now quite difficult to quantitatively predict parameters such as coalescence times. There are several reasons for this, such as the non-reproducibility of experimental data and the fact that describing accurately the film drainage with both interfacial deformation and Marangoni effect is quite complex. But the main problem when describing coalescence is to predict the film thickness at which film rupture will effectively happen, because at length scales of few nanometers there are several physical phenomena which may interfere to either favor or oppose to coalescence. Moreover, when the film thickness is very small, a slight variation of thickness results in a huge variation of the drainage rate and thus on the overall coalescence time. Therefore, the coalescence time is extremely sensitive to the film thickness at rupture, and this is why it is so difficult to quantitatively predict coalescence times even when using sophisticated film drainage models.

In this paper, we are interested in the case where there are micro-particles at the interface. Although this has not been much studied in the past, it is a crucial point in the way of a practical understanding of coalescence since in practice there are always micro-particles in real systems. Following de Gennes [21] dirt particles are probably the main responsible for coalescence occurrence in non-ultra pure conditions (i.e. in most cases). The presence of these micro-particles changes quite importantly the mechanism of coalescence because film rupture may happen when the film thickness becomes comparable to the size of the micro-particles due to a bridging mechanism. This mechanism is well known from antifoaming studies such as Garret [22], Dippenaar [23,24] and Frye and Berg [25] and it is linked with the angle of contact of the interface with the particle surface: a micro-particle which preferentially wets the drop phase will in general reduce the coalescence time by causing the rupture of the film when it reaches a thickness comparable to the particle size.

In the present work, we seek a better understanding of the drop/interface coalescence induced by a bridging mechanism in presence of micro-particles at the interface and contaminants as well. Micro-particles are expected to modify coalescence dynamics through a bridging mechanism and contaminants are expected to tune this mechanism through their effect on interfacial tension and contact angle. In Section 2, we describe the experimental procedure and the measurement techniques. Results are presented and discussed in Section 3 and summarized in Section 4.

\section{Experimental}

\subsection{Experimental setup and protocol}

The experiment has been designed to visualize the coalescence of a water drop settling through a less dense oil towards a plane oil-water interface. Experiments have been performed with and without micro-particles trapped at the oil/water interface. The experimental setup is schematized in Fig. 1. It is located in a room where temperature is regulated at $20^{\circ} \mathrm{C}$. The test section consists 


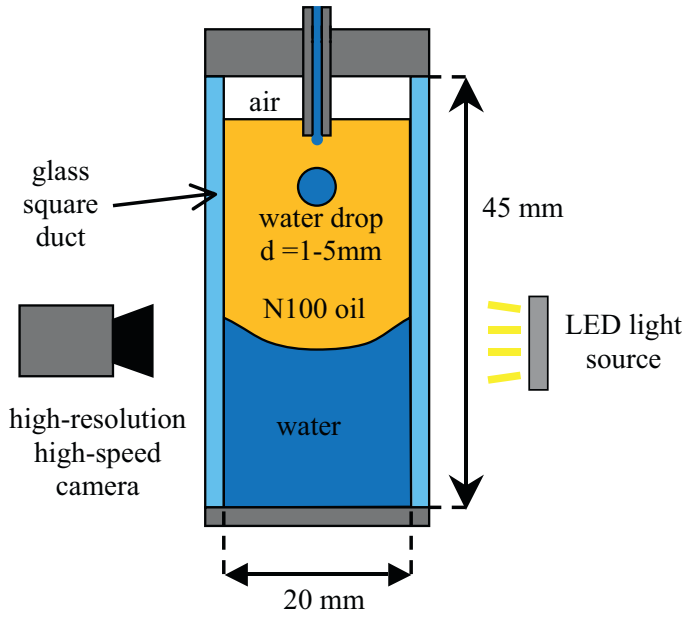

Fig. 1. Experimental setup.

of a glass square duct of $45 \mathrm{~mm}$ height and $20 \mathrm{~mm}$ width, both ends of which are hermetically sealed by two stainless steel plates. It is filled with the water and oil phases with only a small air volume at the top. A small capillary connected to a syringe filled with water is introduced in the oil phase through a small hole located at the center of the top plate. The capillary hermetically seals the hole through which it is introduced but can easily slide through it. Pendant water drops of different volumes are produced manually at the capillary tip using the syringe, and are then detached manually by shaking vertically the capillary. This method enables to produce water drops with diameters ranging from 1 to $5 \mathrm{~mm}$ using a single capillary.

The oil phase is a standard oil viscosity-reference, N100 from Paragon Scientific Ltd.; its density and dynamic viscosity as given by the manufacturer, are respectively $\rho_{0}=865 \mathrm{~kg} \mathrm{~m}^{-3}$ and $\mu_{\mathrm{o}}=283.6 \mathrm{mPas}$ at $20^{\circ} \mathrm{C}$. The water used is distilled water; its density and dynamic viscosity are respectively $\rho_{\mathrm{w}}=998 \mathrm{~kg} \mathrm{~m}^{-3}$ and $\mu_{\mathrm{w}}=1.0 \mathrm{mPa}$ a at $20^{\circ} \mathrm{C}$. The density difference is therefore $\Delta \rho=\rho_{\mathrm{w}}-\rho_{\mathrm{o}}=132 \mathrm{~kg} \mathrm{~m}^{-3}$ and the viscosity ratio $\mu_{\mathrm{w}} / \mu_{\mathrm{o}}=3.5 \times 10^{-2}$. Interfacial tension between water and $\mathrm{N} 100$ oil, measured by means of a pendant-drop tensiometer (Krüss DSA100), is $\sigma_{\text {ow }}=35.3 \mathrm{mN} \mathrm{m}^{-1}$ at $20^{\circ} \mathrm{C}$.

The composition of the N100 oil used in this study is a mixture of mineral oils of different length chains but of unknown composition. Its initial interfacial tension with water $(\sim 35 \mathrm{mN} / \mathrm{m})$ indicates that it possesses some polar compounds, compared to the limit of interfacial tension of $n$-alcane with water $(\sim 52 \mathrm{mN} / \mathrm{m})$.

The micro-particles are hollow glass spheres usually used as particle image velocimetry tracers with a narrow size distribution centred around a mean diameter $d_{\mathrm{p}}$ of $10 \mu \mathrm{m}$ with a r.m.s. value equal to $4 \mu \mathrm{m}$. Particle density $\rho_{\mathrm{p}}$ is of approximately $1200 \mathrm{~kg} / \mathrm{m}^{3}$, which means that they are denser than both liquid phases so their apparent weight is always directed downwards. The condition for them to float at the oil/water interface is the existence of an upward capillary force resulting from the interfacial tension balance at the contact line. Glass particles are hydrophilic, so the contact angle of water is expected to be smaller than $\pi / 2$. In this case, the interfacialforce vertical component can oppose the particle weight only when the contact line is above the equator plane (see also scheme of Fig. 12).

When particles are much smaller than the capillary length (Bond number « 1 ), the knowledge of the contact angle is not crucial to determine whether or not they will float, because it can be shown that they will float even with are very small contact angle. Indeed, for a given density difference, the minimum contact angle of water with a spherical particle $\theta_{c \min }$ that allows a particle to float at an oil-water interface, has been shown by Vella et al. [26] to scale as:

$$
\begin{aligned}
\cos \theta_{\mathrm{c} \text { min }} & =1-\frac{1}{3}\left[\frac{P_{\rho}-P_{\mathrm{o}}}{P_{\mathrm{w}}-P_{\mathrm{o}}}\right] \text { Bo where Bo } \\
& =\frac{\left(P_{w}-P_{0}\right) \mathrm{gd}_{\mathrm{p}}^{2}}{\sigma_{\mathrm{ow}}} \text { is the Bond number }
\end{aligned}
$$

In the present system, the relative density difference $\frac{\rho_{\rho}-\rho_{0}}{\rho_{\mathrm{w}}-\rho_{\mathrm{o}}} \sim 2.5$ and Bo $\sim 10-5$ for $d p=10 \mu \mathrm{m}$, so the minimum contact angle is close to zero. In other words, since a zero contact angle is never observed for real surfaces, these $\mu$-particles will float most of the time as long as the Bond number remains much smaller than 1 (they can even float on a water-air interface). It must be pointed out that this statement is true at equilibrium for a static particle over an interface at rest. A small motion of the particle at the interface may prevent the establishment of this static equilibrium. As further discussed in this section, particles are brought to the interface through a coalescence process of water drops loaded with particles with the oil-water interface. The deposit of particles on the interface results from the film retraction and also from the entrainment of particles by the water flow generated by coalescence, which brings particles from the drop bulk in contact with the interface. Some $\mu$-particles are retained at the interface and some are not, but their retention is driven by the complex dynamics of film retraction, not by the static equilibrium of flotation or wetting considerations.

Before each experimental campaign, the components of the experimental device are disassembled and individually carefully washed by using distilled water, acetone, $n$-heptane and toluene to remove all residual traces of oil from previous experimental campaigns. The components are then dried and reassembled. The test section is first partially filled with distilled water and the N100 oil is then slowly poured in order to prevent the formation of small drops into one or the other phase. The room where the experiments are carried out is not a clean room and the presence of dust particles at the oil-water interface is impossible to prevent when manipulating the fluids during the filling of the cell or the successive refills of the syringe during a campaign.

Because the contact angle of the oil/water interface with the walls is less than $\pi / 2$ (hydrophilic material) and the capillary length $\left(l_{\text {cap }}=\left(\sigma_{o w} /(\Delta \rho g)\right)^{1 / 2} \approx 5.2 \mathrm{~mm}\right.$, with $g$ the gravity acceleration $)$ is not negligible compared to the test section width $(20 \mathrm{~mm})$, the whole interface adopts a 3D shape that is curved upwards. However, even if 3D deformation is quite important near the corners, the shape can be considered axisymmetric and its curvature is small compared to the drop diameters close to the axis, where the drops settle and coalesce. Hereinafter, the interface between the water and oil bulk phases in the cell will be referred to as the "oil/water interface", while the interface between the water drop and the oil will be referred to as the "oil/drop interface".

After the experimental setup has been prepared, each test run proceeds as follows. First, a given volume $\mathrm{V}$ of water is injected manually using the syringe and results in a visible pendant drop attached to the capillary. Detached drops with equivalent diameters $d=(6 \mathrm{~V} / \pi)^{1 / 3}$ ranging between 1 and $5 \mathrm{~mm}$, have been grouped in three classes: small $(d<2.5 \mathrm{~mm})$, medium $2.5 \leq d \leq 4.4 \mathrm{~mm}$ and large $d>4.4 \mathrm{~mm}$. Because the $\mathrm{N} 100$ oil is very viscous and the ratio $\rho_{\mathrm{w}} / \rho_{\mathrm{o}}$ is close to 1 , each drop quickly reaches its terminal velocity, ranging between $0.3 \mathrm{~mm} \mathrm{~s}^{-1}$ for the smallest drops and $3 \mathrm{~mm} \mathrm{~s}^{-1}$ for the largest ones. Maximum value of particle Reynolds number $\left(\operatorname{Re}_{p}=\rho_{0} v_{\infty} d / \mu_{0}\right.$, where $v_{\infty}$ is the drop terminal speed) is less than $5 \times 10^{-2}$ and maximum value of Bond number $\left(\Delta \rho \operatorname{gd}^{2} / 4 \sigma_{\text {od }}\right)$ is 0.22 . In these conditions, drops remain spherical while settling.

The settling of the water drop in the oil phase, its impact with the oil/water interface, and the coalescence process are recorded 


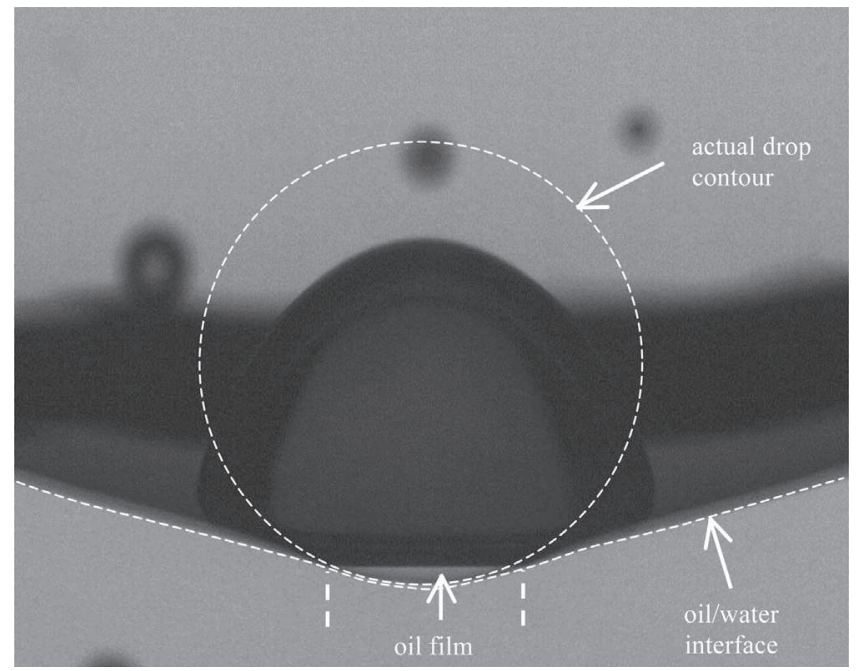

Fig. 2. Typical image of a drop (campaign $\mathrm{nP}$ ) resting on the oil/water interface. (The dash line shows the actual drop contour as it would appear in the absence of optical distortion).

using a PCO DIMAX high-speed camera, allowing a grabbing rate of $1279 \mathrm{fps}$ at high resolution $(2016 \times 2016$ pixels $)$. As a general rule, the camera is carefully positioned perpendicularly to one of the sides of the test section. However, in a few test runs, the camera was tilted forward and placed above the level of the oil/water interface in order to film the coalescence process from above. A LED panel was used as a light source to provide a uniform, intense and stable lighting and avoid the heating of the test section. It was placed at the opposite side of the camera. The drop settling is recorded at low frequency (5 to $100 \mathrm{fps}$ ) in order to accurately measure the drop diameter and terminal velocity. As the drop approaches the oil/water interface, it decelerates while the interface deforms. Eventually, the drop reaches the interface and rests over it with no apparent internal or external motion, which corresponds to the moment when the chronometer is started. The camera is then set to a high-speed rate (from 500 to $4000 \mathrm{fps}$ ) in order to capture the hole expansion dynamics during coalescence. The drop may rest over the interface from minutes to hours until coalescence takes place. Right after it has occurred, the chronometer and the camera are stopped (only the last thousand images are saved, ensuring that the film rupture has been shot). A new drop is then formed.

Pictures of a drop resting over the oil/water interface are displayed in Figs. 2 and 3 and. Images are distorted because the drops are viewed through the meniscus formed by the oil/water interface with the walls of the container. For drops with a diameter larger than the meniscus height, the top part of the drop appears undistorted (Fig. 3b). However, it has been checked that the actual shape of a resting drop (represented by a dashed line in Fig. 2) remains actually nearly spherical. The maximal deformation is observed for the largest drops $(d \approx 4.9 \mathrm{~mm})$ and corresponds to a drop height that is $10-15 \%$ smaller than the drop equivalent diameter, which is measured during the drop fall when it is spherical.

Resting drops show two clear distinct zones: a large dark zone in the main part of the drop from the top to the bottom and a smaller and lighter zone at the bottom near the interface. The lower zone is in contact with a thin oil film where a Stokes flow likely develops during the drainage process [27]. Because this oil film is very thin, the optical deformation induced by the refractive index difference between water and oil is minimized in this zone. In the upper zone, the water and the surrounding oil are at rest and the pressure is hydrostatic. The frontier between these two zones is horizontal and extremely sharp, suggesting that the thickness of the oil film suddenly diverges at the rim. It can be concluded that there is a very sharp transition between the Stokes flow region and the hydrostatic region. Note that even if using fluids with close refractive indices minimizes optical distortion [7-9], the current system of liquids with contrasted optical indices allows an accurate detection of the boundary of the thin oil film at the interface.

Two main experimental campaigns have been carried out. Neither the water nor the oil initially present in the test section was replaced during an experimental campaign. Therefore, the water volume, and thus the interface level varied a little bit during a campaign. However, the oil/water interface always remained approximately in the middle of the test section, and most importantly, it always remained sufficiently far from the capillary tip so that the detached drops were always able to reach their terminal velocity before approaching the interface. For both campaigns, small, medium and large drops were alternatively injected in order to analyze the role of the drop size at various ages of the oil/water interface. Campaign nP is the reference case and corresponds to the experiments carried out without the addition of micro-particles, where 151 drops of water were successively released during a period of 14 days.

Campaign P investigated the influence of glass micro-particles trapped at the water-oil interface, where a sample of 76 drops was released over the same period of time (14 days). The experimental procedure for campaign $P$ was the following: during the first 5 days, distilled water drops loaded with micro-particles were injected in the cell. After each coalescence, a small amount of the particles contained in the drop ended up trapped at the oil/water interface, while the rest settled in the bottom of the test section. This observation confirms the a priori analysis of particle flotation condition at the interface. At the end of the fifth day, a noticeable amount of trapped particles was distributed over the entire oil/water interface. Then, after a day without drop injections, only water drops without particles were injected in the cell from the seventh day, but their coalescence was investigated in the presence of previously collected micro-particles at the oil/water interface.

A typical sequence of the film rupture during the coalescence of a large drop is shown in Fig. 4. Depending on the field size, the camera resolution varies between $90 \mathrm{px} / \mathrm{mm}$ and $150 \mathrm{px} / \mathrm{mm}$. Hence, micro-particles represent one or two pixels, just enough to be detected on the images. In this sequence, the hole expansion through the oil film is clearly visible. The onset of the hole formation may occur anywhere in the oil film, in the visible front part of the drop as in Fig. 4, as well as in the opposite side of the drop. In the latter case, the hole is still visible through the front portion of the oil film, but the image is then of lower quality.

In each experiment, drop diameter and terminal velocity are measured during the drop settling from video acquisition at a low frame rate (from 5 to $100 \mathrm{fps}$ ). The time spent by a drop settling over the oil/water interface prior to coalescence is measured by means of a chronometer with an accuracy of one second, which is good enough since drop resting time on the interface ranges from minutes to hours. The interfacial tension of the oil/water interface is measured in the case of large drops, from shape analysis of the oil/water interface, which deforms under the action of drop apparent weight (see Fig. 3). Finally, the retraction speed of the film during coalescence is measured using high-speed movies (up to $4000 \mathrm{fps}$ ).

\subsection{Determination of surface tension at the oil/water interface}

Fig. 3 shows the pictures of two drops resting over the oil/water interface during the campaign nP. The first was taken during the first day, while the second was taken on day 13. Even if both drops have an equal size, curvature of oil/water interface is more pronounced in the case of the older interface (Fig. 3b). Because the fluids that fill the test section are not renewed, each injected 


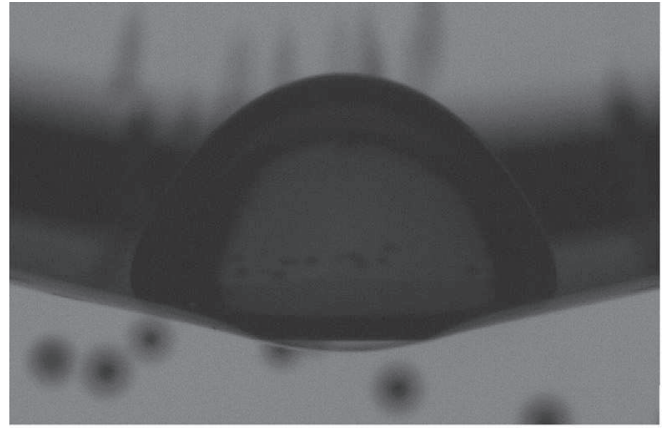

(a)

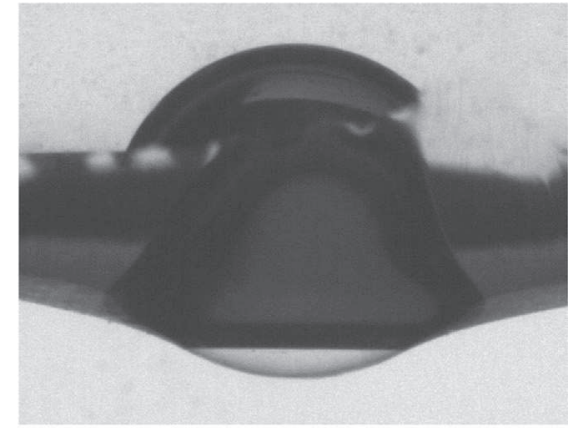

(b)

Fig. 3. Images of two drops of the same size (large drops of Campaign nP) at two different oil/water interface ages: (a) day 1 ; (b) day 13.
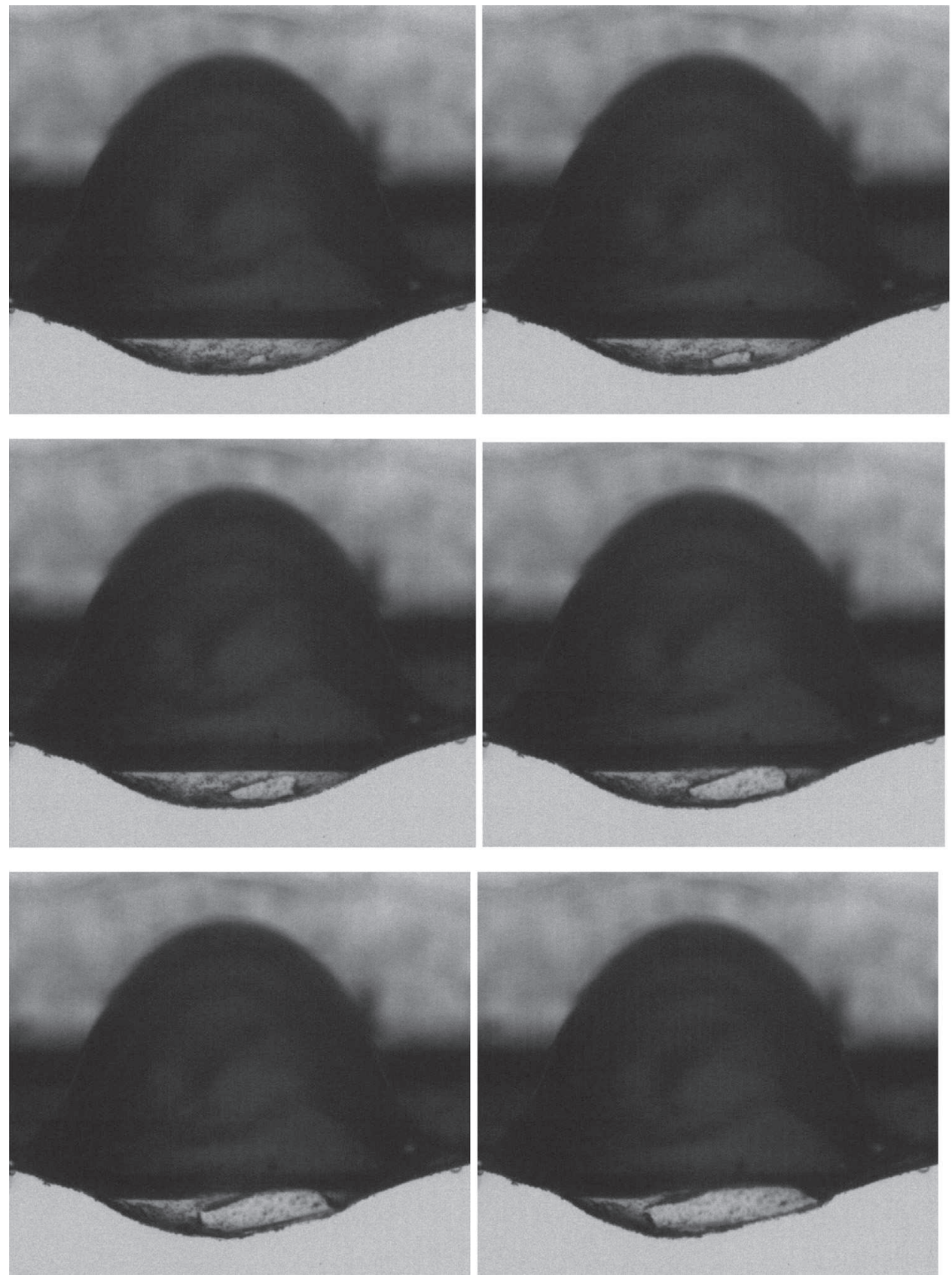

Fig. 4. Typical sequence of the hole expansion during the coalescence of a large drop in the presence of micro-particles trapped at the oil/water interface (Campaign P). The time interval between two successive images is $1 \mathrm{~ms}$. 


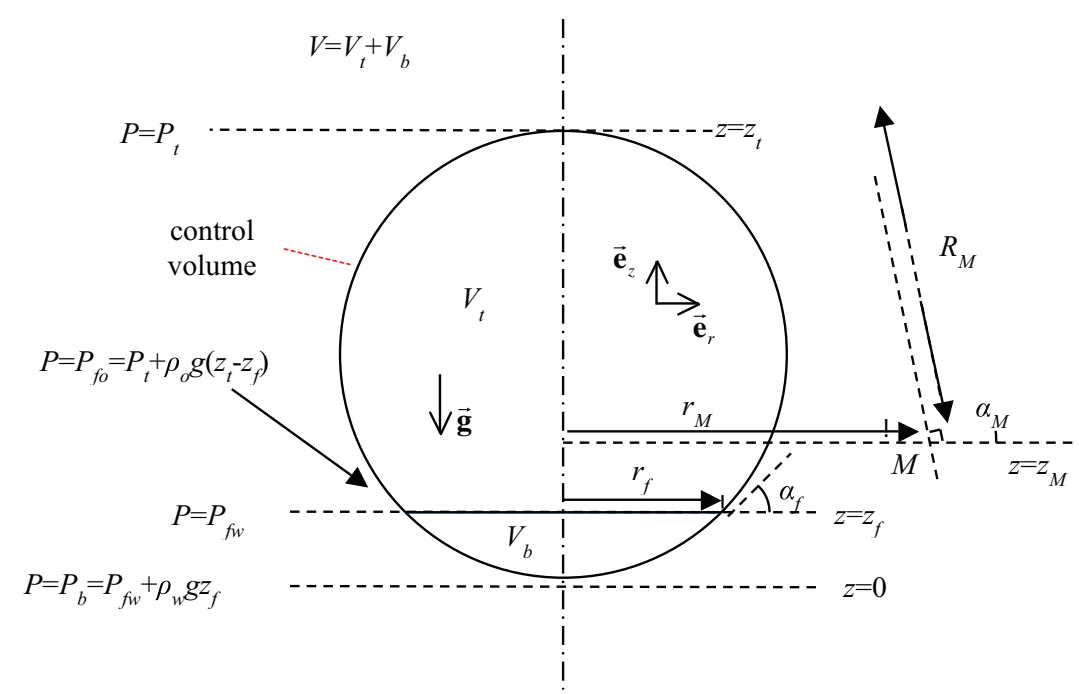

Fig. 5. Schematic drawing of a drop settling over the oil/water interface.

drop progressively contaminates the oil/water interface, even if the contamination of each individual drop is low. Consequently, the interfacial tension $\sigma_{\text {ow }}$ of the oil/water interface decreases day after day, during a given campaign. The value of $\sigma_{\text {ow }}$ can be directly determined within the test section by considering the deformation of the oil/water interface caused by a drop resting over it. The method is described below.

We consider an axisymmetric resting drop as schematized in Fig. 5 . The symmetry plane is defined by unit vectors $\left(\vec{e}_{\mathrm{r}}, \vec{e}_{\mathrm{z}}\right)$ in a cylindrical coordinates frame $(r, \theta, z)$. We set $z=0$ at the oil/water interface. The resting drop is split into a bottom volume $V_{\mathrm{b}}$ that corresponds to the zone in contact with the thin oil film, and a top volume $V_{\mathrm{t}}$ in contact with the hydrostatic oil region above. The surface separating these two volumes is a horizontal disk of radius $r_{\mathrm{f}}$ located at $z=z_{\mathrm{f}}$. The tangent to the oil/water interface at $z_{\mathrm{f}}$ makes an angle $\alpha_{\mathrm{f}}$ relative to the horizontal direction.

The hydrostatic pressure in the oil phase at the top of the drop $\left(z=z_{\mathrm{t}}\right)$, is noted $P_{\mathrm{t}}$, while the hydrostatic pressure in the water phase at the bottom of the oil/water interface is noted $P_{\mathrm{b}}$. Hydrostatic pressures at $z=z_{\mathrm{f}}$ are respectively noted $P_{\mathrm{fw}}$ in the water phase and $P_{\text {fo }}$ in the oil phase. It hence yields:

$P_{\text {fo }}=P_{t}+\rho_{\mathrm{o}} g\left(z_{\mathrm{t}}-z_{\mathrm{f}}\right)$

and

$P_{\mathrm{fw}}=P_{\mathrm{b}}-\rho_{\mathrm{w}} g z_{\mathrm{f}}$

We consider the force balance on the control volume delimited by the red dotted line in Fig. 5, which surrounds the upper volume $V_{t}$, crosses the oil/water interface at $z=z_{\mathrm{f}}$, and then surrounds the lower volume $V_{\mathrm{b}}$. In the vertical direction, it reads:

$F_{\mathrm{tz}}+F_{\mathrm{bz}}-\rho_{\mathrm{w}} v_{\mathrm{g}}+2 \pi r_{f} \sin \left(\alpha_{\mathrm{f}}\right) \sigma_{\mathrm{ow}}=0$

where $F_{\mathrm{tz}}$ and $F_{\mathrm{bz}}$ are respectively the resultants of the hydrostatic pressure forces exerted on the control volume by the oil phase on the top and by the water phase on the bottom.

Note that when $\mu$-particles are present, the contribution of the weight of the $\mu$-particles and that of the variation of the interface area due to their presence can be neglected in the force balance (3).

To evaluate $F_{\mathrm{tz}}$, we consider that a volume $V_{\mathrm{t}}$ that would be totally immersed in a hydrostatic oil phase with the same hydrostatic pressure $P_{t}$ at its top. The resultant of the pressure forces exerted by the oil phase is then the buoyancy force, which is the sum of the force exerted by the oil on the upper part of $V_{\mathrm{t}}$ and the force exerted by the oil on the flat bottom surface of $V_{\mathrm{t}}$ :

$F_{\mathrm{tz}}+\pi r_{\mathrm{f}}^{2} P_{\mathrm{fo}}=\rho_{\mathrm{o}} V_{\mathrm{t}} \mathrm{g}$

The same method can be used to evaluate $F_{\mathrm{bz}}$ by considering the volume $V_{\mathrm{b}}$ :

$F_{\mathrm{bz}}-\pi r_{\mathrm{f}}^{2} P_{\mathrm{fw}}=\rho_{\mathrm{w}} V_{\mathrm{b}} \mathrm{g}$

Eq. (3) can be recast using Eqs. (4) and (5):

$2 \pi r_{\mathrm{f}} \sin \left(\alpha_{\mathrm{f}}\right) \sigma_{\mathrm{ow}}-\pi r_{\mathrm{f}}^{2}\left(P_{\mathrm{fo}}-P_{\mathrm{fw}}\right)=\left(\rho_{\mathrm{w}}-\rho_{\mathrm{o}}\right) g\left(V-V_{\mathrm{b}}\right)$

This equation relates $\sigma_{\text {ow }}$ to the geometric parameters $\alpha_{\mathrm{f}}, r_{\mathrm{f}}, V$ and $V_{\mathrm{b}}$. The angle $\alpha_{\mathrm{f}}$ and the radius $r_{\mathrm{f}}$ are directly measured on an image of a resting drop. The drop volume $V$ is measured during the drop fall before the interaction with the oil/water interface. The bottom volume $V_{\mathrm{b}}$ is determined from an image of the resting drop. The interface contour from $z=0$ to $z=z_{\mathrm{f}}$ is fitted by a 4th-order polynomial: $z=f(r)=a_{4} r^{4}+a_{2} r^{2}$ with $f\left(r_{\mathrm{f}}\right)=z_{\mathrm{f}}$ and $\mathrm{df} / \mathrm{d} r\left(r_{\mathrm{f}}\right)=\tan \left(\alpha_{\mathrm{f}}\right)$. After integration, it yields:

$V_{\mathrm{b}}=\frac{1}{3} \pi r_{\mathrm{f}}^{2} z_{\mathrm{f}}+\frac{1}{12} \pi r_{\mathrm{f}}^{3} \tan \left(\alpha_{\mathrm{f}}\right)$

The pressure jump through the oil/water interface at $z=z_{\mathrm{f}}$, $P_{\mathrm{fo}}-P_{\mathrm{fw}}$, is related to the interface curvature. However, a precise measurement of this curvature based on the camera images is difficult. Therefore, $P_{\mathrm{fo}}-P_{\mathrm{fw}}$ is determined as follows. We consider $\mathrm{M}$, the inflexion point of the intersection of the oil/water interface with the plane of symmetry. The coordinates $\left(r_{\mathrm{M}}, z_{\mathrm{M}}\right)$ of M can be accurately determined from the images by measuring the evolution of the inclination angle of the oil/water interface. The inflexion point corresponds to a minimal or maximal value $\alpha_{\mathrm{M}}$ of this angle. At M, the curvature of the interface reduces to that which is perpendicular to the plane of symmetry, which can be accurately measured, and the pressure jump through the interface reads:

(8) $P_{\mathrm{o}}(\mathrm{M})-P_{\mathrm{w}}(\mathrm{M})=\frac{\sigma_{\mathrm{ow}}}{R_{\mathrm{M}}}$, where the curvature radius $R_{\mathrm{M}}$ is given by the distance between $M$ and the intersection of the axis of symmetry with the normal to the interface at $\mathrm{M}$,

$R_{\mathrm{M}}=\frac{r_{\mathrm{M}}}{\sin \left(\alpha_{\mathrm{M}}\right)}$ 
The pressure at $z=z_{\mathrm{f}}$ is related to the pressure jump at $z=z_{\mathrm{M}}$ through the difference of hydrostatic pressure between these two points:

$P_{\mathrm{fo}}-P_{\mathrm{fw}}=\frac{\sigma}{R_{\mathrm{M}}}-\left(\rho_{\mathrm{w}}-\rho_{\mathrm{o}}\right) g\left(z_{\mathrm{M}}-z_{\mathrm{f}}\right)$

The following expression for $\sigma_{o w}$ is finally obtained:

$\sigma_{\mathrm{ow}}=\frac{\left(\rho_{w}-\rho_{\mathrm{o}}\right) g\left[V-V_{\mathrm{b}}-\pi r_{\mathrm{f}}^{2}\left(z_{\mathrm{M}}-z_{\mathrm{f}}\right)\right]}{2 \pi r_{\mathrm{f}} \sin \left(\alpha_{\mathrm{f}}\right)-\pi r_{\mathrm{f}}^{2} / R_{\mathrm{M}}}$

The more deformed the oil/water interface (larger drop or lower interfacial tension), the better is the accuracy in the determination of $\sigma_{\text {ow }}$. That is the reason why we have used this method only in the case of large drops $(d>4.4 \mathrm{~mm})$. The resulting accuracy is of the order of $\pm 1 \mathrm{mN} \mathrm{m}^{-1}$ in the first days of a campaign when the interfacial tension is close to the initial value of a fresh $\mathrm{N}-100 /$ water interface $\left(\sigma_{\mathrm{ow}}=35-40 \mathrm{mN} \mathrm{m}^{-1}\right)$, and less than $\pm 0.3 \mathrm{mN} \mathrm{m}^{-1}$ at the end of a campaign, when the interface becomes significantly contaminated $\left(\sigma_{\mathrm{ow}}=10-15 \mathrm{mN} \mathrm{m}^{-1}\right)$. Note that this method relies on the same principle as the pendant drop method and possesses the same limitations (a sufficient gradient of curvature along the interface is required) and the same level of accuracy. However, compared to the pendant drop technique, it's an in situ nonintrusive measurement that allows to measure the surface tension of the planar oil-water interface as it is aging over long times.

\subsection{Measurement of film retraction speed}

The coalescence process is filmed at a frequency ranging from 500 to $4000 \mathrm{fps}$ (Fig. 4). The initiation of coalescence occurred at random locations, although more frequently in the vicinity of the oil film rim (probably due to the formation of a dimple). In our experiment, the coalescence of drops is observed from a side view (although ideally the film retraction would have been better visualized from a bottom view as it was done in Charles and Mason [6]. Since the vertical extension of the oil film is small, it could be only properly observed for large drops $(d>4 \mathrm{~mm})$. Therefore, as in the case of surface tension, retraction speed was measured only for larger drops.

In all experiments, the oil film is curved. However, at the beginning of the retraction process, the hole radius is quite small compared to the drop radius. For that reason, the retraction speed was measured from the first images of the hole development sequence, i.e. when the film can be still considered as being plane. A difficulty arises from the fact that the retraction plane, i.e. the plane tangent to the oil/water interface at the point of film-rupture onset, does not necessarily coincide with the image plane, and the hole shape may appear elongated due to a perspective effect. Assuming that the oil/water interface is axisymmetric and using the polynomial fit introduced in Section 2.2, it is possible to calculate the 3D coordinates of any point on the interface. In particular, the coordinates of each point of the hole can be derived and the shape of the hole in the retraction plane can be drawn. Fig. 6 shows an example of application of this procedure. While the hole appears quite elongated on the raw image (Fig. 6a), it is approximately circular in the retraction plane (Fig. 6b), even if small and temporary shape irregularities are sometimes observed due to a local accumulation of particles. This procedure, applied to a significant sample of coalescence events, always led to the conclusion that the holes expand in all directions at the same speed.

The retraction speed is measured either from original images or after projection onto the retraction plane. When using original images, perspective effects are limited by considering the horizontal velocity when the initiation point is close to the symmetry axis or when one of the hole edges passes near the symmetry axis. It is particularly well estimated when the hole onset location is close to the oil film curved rim, because the hole radius then corresponds to the length of the portion of the film interface that vanishes. In all test runs, the retraction speed was observed to follow the same trend. It increases fast at the very beginning, during the first 2-3 images at $4000 \mathrm{fps}$, corresponding to a period of approximately $1 \mathrm{~ms}$, but this acceleration cannot be accurately quantified due the reduced size of the hole in the first images. Then, it quickly reaches a constant value, $u_{\text {exp }}$, which is the one that is measured.

The overall accuracy of the measurement of $u_{\text {exp }}$ is rather poor ( $\pm 25 \%$ ), owing to geometrical complications (perspective effects, interface curvature) and detection problems of the hole contour in some cases. However, despite this limitation, it is still possible to investigate the huge variations of retraction speed - more than one order of magnitude - between the beginning and the end of each campaign (nP and $\mathrm{P}$ ).

\section{Results and discussion}

\subsection{Drop settling velocity}

In the whole range of diameter investigated, settling drops are spherical and have reached their terminal velocity before reaching the oil/water interface. Measured terminal velocities are plotted in Fig. 7 as a function of the drop diameter (symbols). Terminal velocities of solid spheres settling in a square cylinder at low Reynolds number from Miyamura et al. [28] are also plotted, as well as terminal velocities of spheres in an unbounded domain (Stokes law, $v_{\infty \text {-unbounded }}=\Delta \rho \mathrm{gd}^{2} / 18 \mu o$ ). Confined spherical particles (solid line) settle at a much slower speed than in an unbounded domain (dashed line). Particles Reynolds number based on measured terminal velocity ranges between $9 \times 810^{-4}$ and $4 \times 610^{-2}$. In Stokes regime, terminal velocity can be predicted as the product of the terminal velocity in an unbounded domain and an increasing function of the ratio $d / L$, where $L$ is the width of the cell in the present case $(L=20 \mathrm{~mm})$. Using that correction factor (polynomial development of $d / L$ ) it can be seen in Fig. 7 that experimental data nicely fit the theoretical prediction of Miyamura et al. [28]. Moreover, if we consider the case of pure fluids, the corrected Hadamard's solution in a spherical confinement, taken from Satapathy and Smith [29], reads:

$v_{\infty \_ \text {unbounded }}=\frac{\Delta \rho g d^{2}}{24 \mu_{o}}(2-3 d L)$ with $\frac{\mu_{\mathrm{w}}}{\mu_{\mathrm{o}}}$

Eq. (12) predicts settling velocity for the present system growing from $0.38 \mathrm{~mm} / \mathrm{s}$ for a $1 \mathrm{~mm}$ diameter drop to $6.2 \mathrm{~mm} / \mathrm{s}$ for a $5 \mathrm{~mm}$ diameter drop. These values coincide with those obtained with spherical particles in an unbounded medium (dashed line), and are therefore not representative of present experimental data. Finally, it was checked that drop deformation (even if very small) could not make deviate the settling velocity in an unbounded domain from Hadamard's law. Based on the asymptotic development of Taylor and Acrivos [30], with the present system (/ «1) the correction to the drag coefficient scales as:

$C_{D}-C_{\text {Dspher }} \cong \frac{6}{5} \mathrm{Ca}$

where $\mathrm{Ca}=\mu_{\mathrm{o}} v_{\infty} / \sigma_{\sigma \mathrm{w}}$, with a maximum value of 0.025 to be compared to $16 / \operatorname{Re}_{\mathrm{p}} \sim 500$. These results confirm that drops remain spherical while settling and their interface behaves as a solid wall, suggesting that it is immobilized by surface-active contaminants.

\subsection{Evolution of interfacial tension at the oil/water interface}

Fig. 8 shows the time evolution of the interfacial tension $\sigma_{\text {ow }}$ of the oil/water interface during campaigns $\mathrm{nP}$ and $\mathrm{P}$. In both cases, initial values are about the same $\left(\sigma_{\mathrm{ow}}=35-40 \mathrm{mN} \mathrm{m}^{-1}\right)$ and then 
(a)

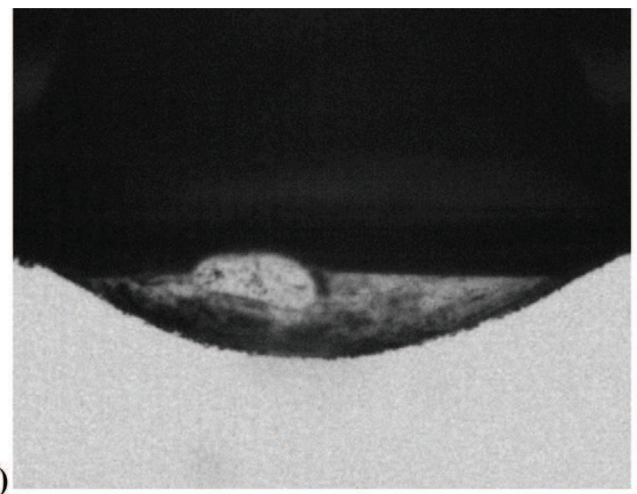

(b)

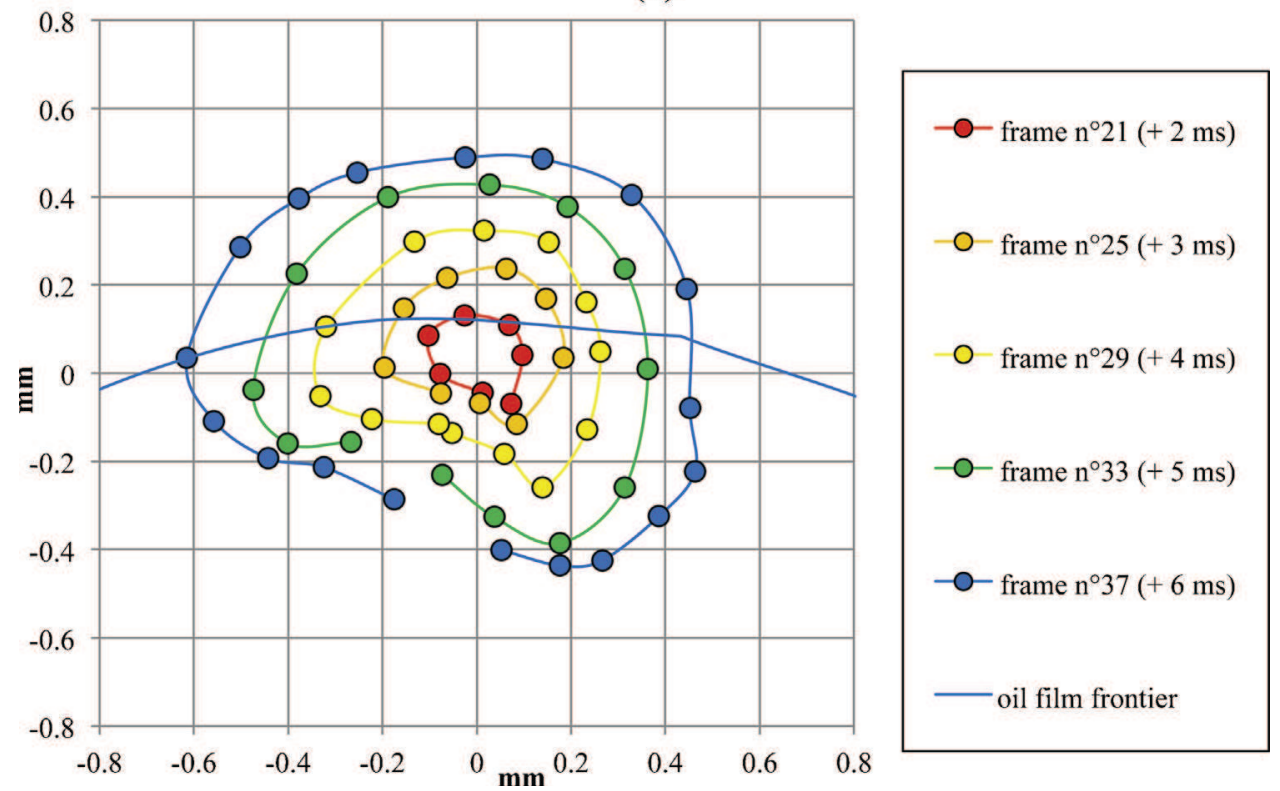

Fig. 6. Evolution with time (cf frame number in the legend) of hole shape in the oil film after film rupture. (a) View in the camera plane (movie frame ${ }^{\circ} 33$ ); (b) projections of the hole contour on the retraction plane after reconstruction.

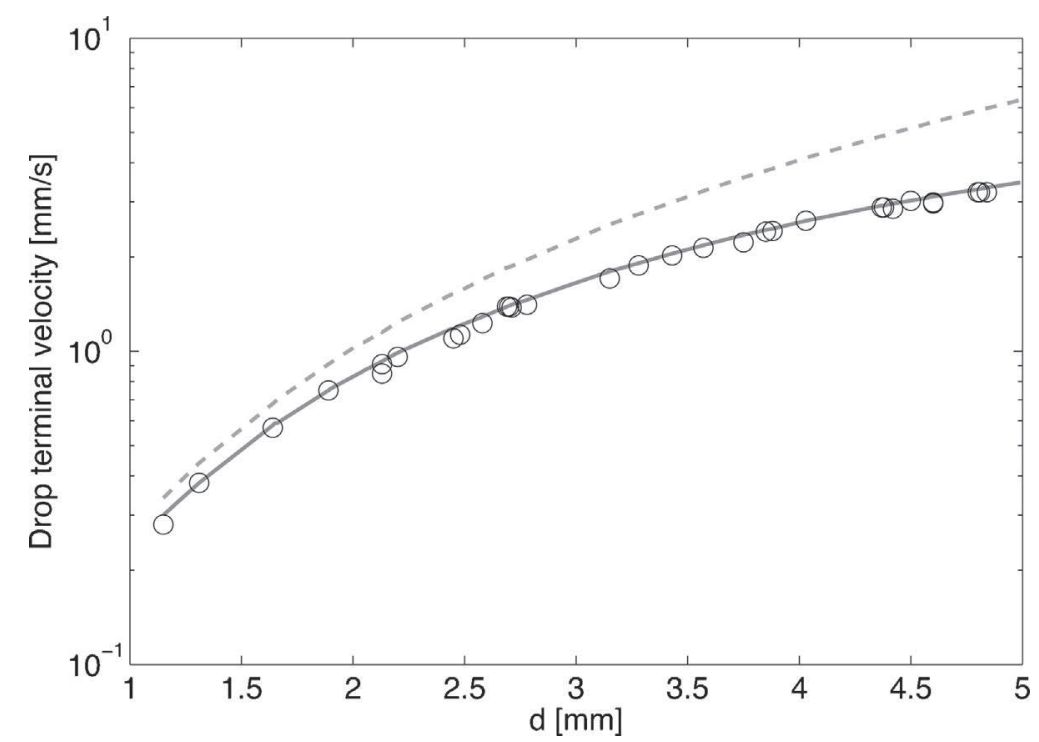

Fig. 7. Terminal velocities as a function of particle diameter: Experimental values (symbols); unbounded spherical particles or Stokes law (dashed line); spherical particles in a square cylinder (plain line) (Miyamura et al. [27]). 


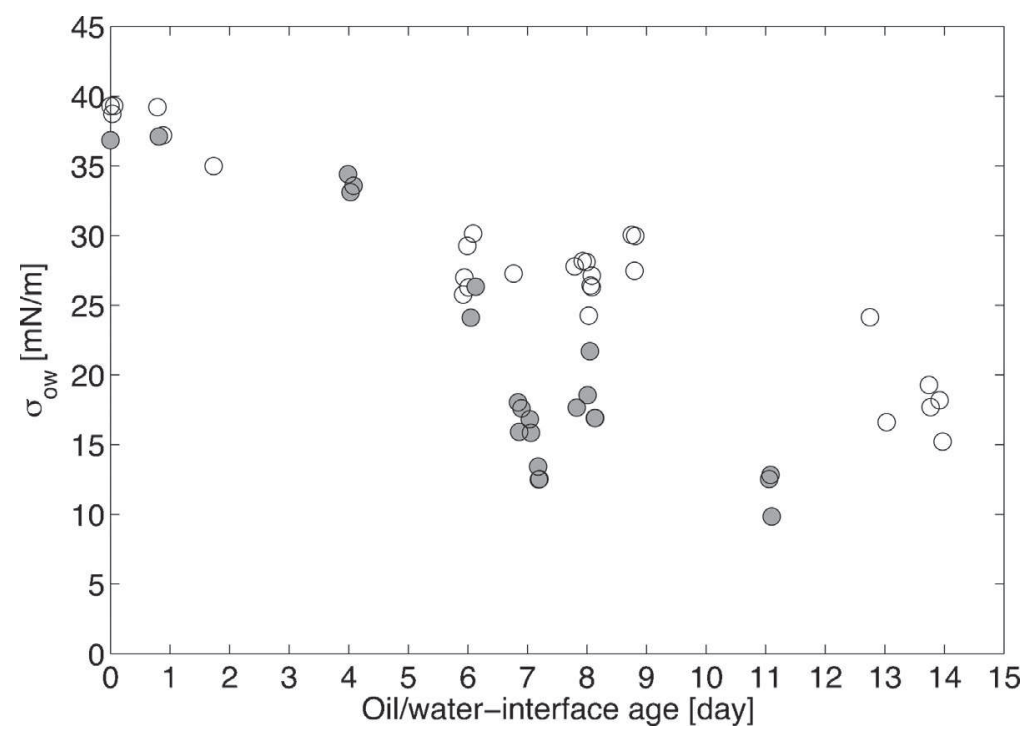

Fig. 8. Evolution of the interfacial tension of the oil/water interface with time. Empty circles: campaign nP; plain circles: campaign P.

decrease over the days, the lower value being attained during the campaign with particles. During the campaign without particles (nP), one observes an overall decrease down to $15.2 \mathrm{mN} \mathrm{m}^{-1}$ after 14 days.

This long time decrease of the interfacial tension originates from the impurities contained in the N100 oil resulting from its production process, most likely traces of amphiphilic long chain molecules (fatty acids, aromatic compounds, etc.) which adsorb at the interface at very long times. This slow adsorption process is due to the fact that most of the time (i.e. between coalescence events), the adsorption is diffusion controlled and that the oil viscosity is high ( $\sim 0.3$ Pa s). Note that due to interphase mass transfer during settling in the oil phase, water drops also feed the interface with the contaminants contained in the N100.

When particles are added (P), a slow decrease, similar to that observed in campaign $\mathrm{nP}$, is first observed for the first 4 days while introducing solid particles, then a stronger decrease takes place during next 4 days and the decrease slows down again until $\sigma_{\text {ow }}$ eventually reaches $9.8 \mathrm{mN} \mathrm{m}^{-1}$. This result suggests that additional contaminants are brought by the particles to the oil/water interface.

\subsection{Drop lifetimes}

We examine now the drop lifetime, which is defined as the time during which a drop is resting above the oil/water interface until film rupture occurs.

Fig. 9a shows the results of the campaign without added particles (nP). One observes a very large random scattering of the measured lifetimes, ranging from $20 \mathrm{~s}$ to $7000 \mathrm{~s}$. Such large variations can occur between consecutive events during a same day and no significant evolution of the lifetime with the interface age is observed, even if a slight decrease of the maximum values can be noticed between the beginning and the end of Campaign nP. Moreover, no general trend related to the various drop sizes is discernable.

Fig. 9b presents the lifetimes measured during Campaign P. The presence of micro-particles trapped at the oil/water interface drastically changes the former experimental trends. The dispersion of the results is reduced and shorter lifetime values are measured. However, the precise picture is somewhat complex. Just after each long period (lunch time, nights or weekends) during which no drop was injected, a jump of lifetime upwards is noticed. Then, for a given diameter, the lifetimes of successive drops are generally observe to decrease even if it can remain constant over periods of one to three hours. Overall, day after day, lifetimes show a net tendency towards decrease. Lifetimes are ranging from $700 \mathrm{~s}$ to $10^{4} \mathrm{~s}$ during the first days, and only range from a few to a hundred seconds on day 14 .

In campaign $\mathrm{P}$, in order to discriminate between the effect of aging of the interface and that of the drop diameter upon the drop lifetime, we have reported in Fig. 10 the measured drop lifetime as a function of the drop diameter for periods during which interfacial tension of oil-water interface remained nearly constant (a period of $2 \mathrm{~h} 30$ on day 6 and one of $1 \mathrm{~h} 30$ on day 7). We observe that drop lifetime is reproducible, and is a clear growing function of drop diameter. This evolution is quite significant since the scattering of the measurements is weak despite the fact that during these time periods, the tests have been done by alternating small, medium and large drop sizes in order to avoid any bias related to a possible variation of interface properties. This suggests that, during periods of time when the properties of the oil/water interface do not evolve significantly, the presence of glass particles makes drop lifetime reproducible whereas it remains otherwise random. Indeed, in campaign $\mathrm{nP}$, such reproducible trends over relatively long time periods (1-2 h) were never observed, whatever the chosen time period, and drop lifetimes are fully random this case.

Fig. 11 shows the evolution of the drop lifetime as a function of the interfacial tension of the oil/water interface. Since the interfacial tension measurement is restricted to the larger drops in order to be accurate, drop diameters in this plot are nearly constant $(4.4 \mathrm{~mm}<\mathrm{d}<4.9 \mathrm{~mm})$. In the absence of added particles, drop lifetimes and interfacial tension are uncorrelated. In the presence of added particles, drop lifetime is observed to be an increasing function of $\sigma_{\text {ow }}$. A significant scatter is however still present, suggesting that interfacial tension is probably not sufficient to fully characterized the interface.

To interpret these data, it is useful to recall the fundamentals of the coalescence process, which can be decomposed into three stages. The first stage takes place when the drop deforms the oil/water interface and causes the formation of a thin film of oil between the drop and the water below. During the second stage, the oil film is slowly drained out while both the drop and the oil/water interface keep an almost constant shape. Third, when it has reached a critical thickness $h_{\mathrm{c}}$, the film ruptures and the hole through it expands very quickly, achieving the coalescence. Since the first 


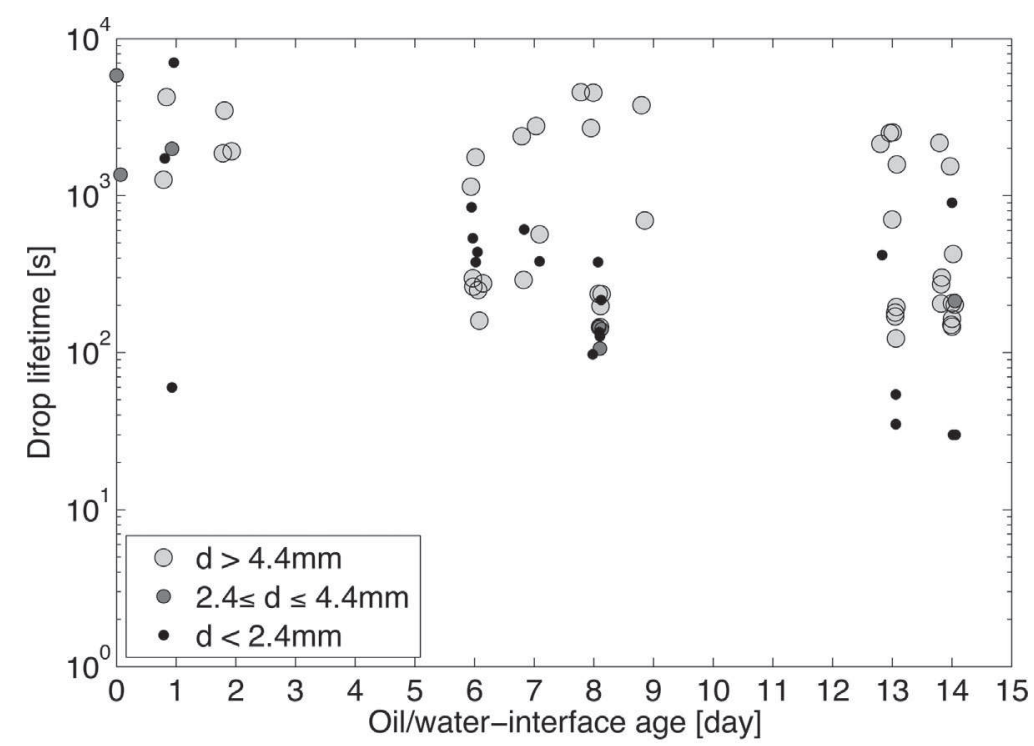

(a)

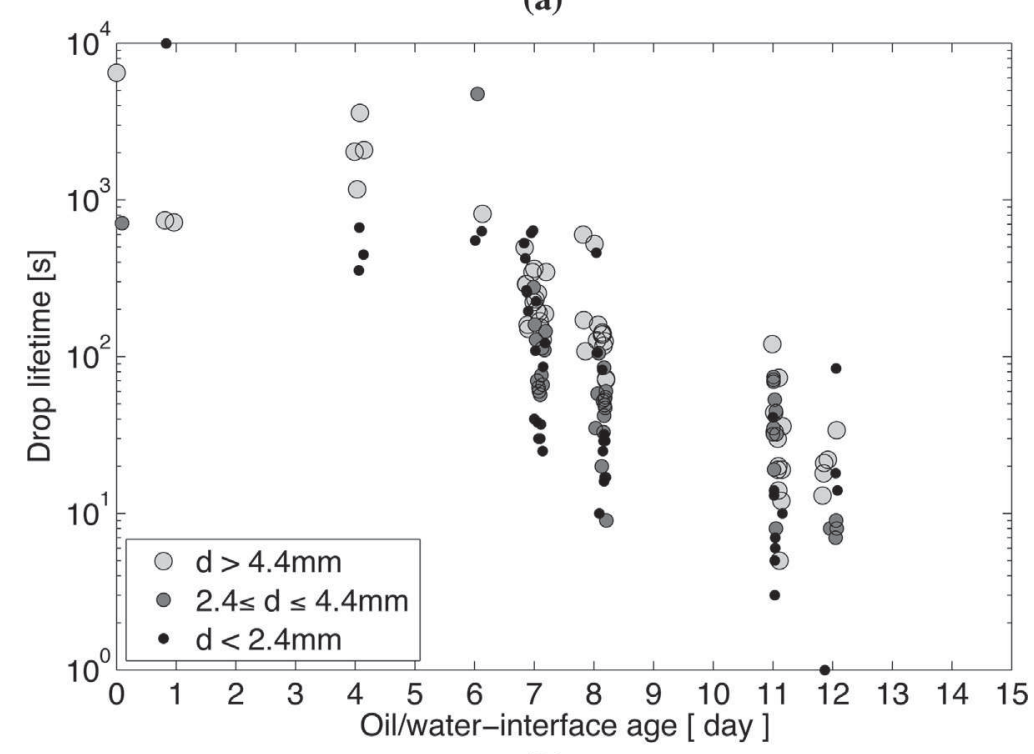

(b)

Fig. 9. Evolution of drop lifetime as a function of time. (a): campaign nP; (b): campaign P.

and the third stages are very short compared to the second one, the drop lifetime is controlled by the drainage time. In order to estimate it, one needs to model the equilibrium shape of the interfaces, the rate of decrease of the oil-film thickness and the value of $h_{\mathrm{c}}$. Such a calculation has been achieved by Lang and Wilke $[15,16]$, assuming that the two facing interfaces have equal and constant interfacial tension as well as a constant radius of curvature. The interface deformation is controlled by the drop Bond number and the drop lifetime is computed assuming a Poiseuille flow in the film (immobile interfaces):

$T_{\mathrm{L}}=\frac{3}{4} \frac{J(\mathrm{Bo}) d^{3} \mu_{o}}{\sigma_{\mathrm{ow}} h_{\mathrm{c}}^{2}}$

where $J(\mathrm{Bo})$ is a growing function of the Bond number $\left(\mathrm{Bo}=\Delta \rho \mathrm{gd}^{2} / 4 \sigma_{\mathrm{ow}}\right)$. This model hence predicts that $T_{\mathrm{L}}$ is an increasing function of $\mathrm{d}$ and a decreasing function of $\sigma_{\text {ow }}$. Note that this model accounts for the presence of surfactants by considering that the interfaces are immobilized (adhesion condition at the film interface) although a more complex Marangoni effect may occur, capable of generating flow velocity in the film directed towards the film center [31,32].

If the interface is free of contaminants, the low value of the viscosity ratio shifts the Poiseuille flow in the film towards a plug flow controlled by the viscosity of the drop phase [27]. Assuming a Stokes flow in the film and equal interfacial tension on both film interfaces, the use of an approximate solution of the drainage time as a function of film thickness as proposed by Chesters [33] or Yiantsios and Davis [34] is adequate in the present case, leading to (in the case of a flat film):

$T_{L} \cong \sqrt{\frac{2}{3}} \operatorname{Bo}^{1 / 2} \frac{\mu_{\mathrm{w}} d^{2}}{\sigma_{\mathrm{o}} h_{\mathrm{c}}}$

Compared to Eq. (14), it is clear that the mobile interface regime (Eq. (15)) predicts much shorter coalescence times, with a factor of the order of $h_{\mathrm{c}} \mu_{\mathrm{w}} /\left(d \mu_{\mathrm{o}}\right)=O\left(10^{-7}\right)$.

Given a value of $h_{\mathrm{c}}$, we can therefore estimate the lifetimes for clean interfaces $\left(\sigma_{\mathrm{ow}}=35.3 \mathrm{mN} \mathrm{m}^{-1}\right)$. Assuming that the film drains until attractive van der Waals molecular forces cause it to rupture, 


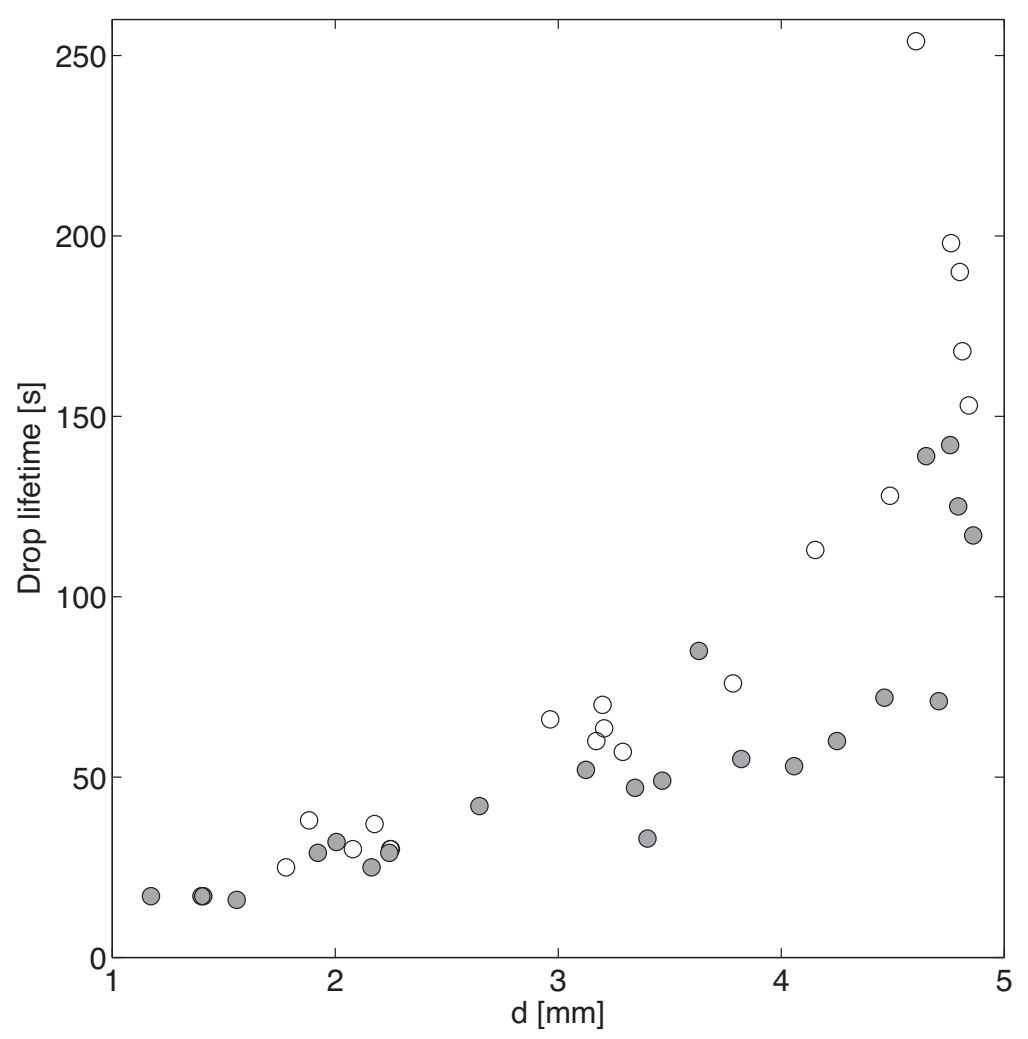

Fig. 10. Drop lifetime versus drop diameter during two short periods of campaign P. Empty circles: a period of 2 h 30 on day 6 ; plain circles: a period of 1 h 30 on day 7.

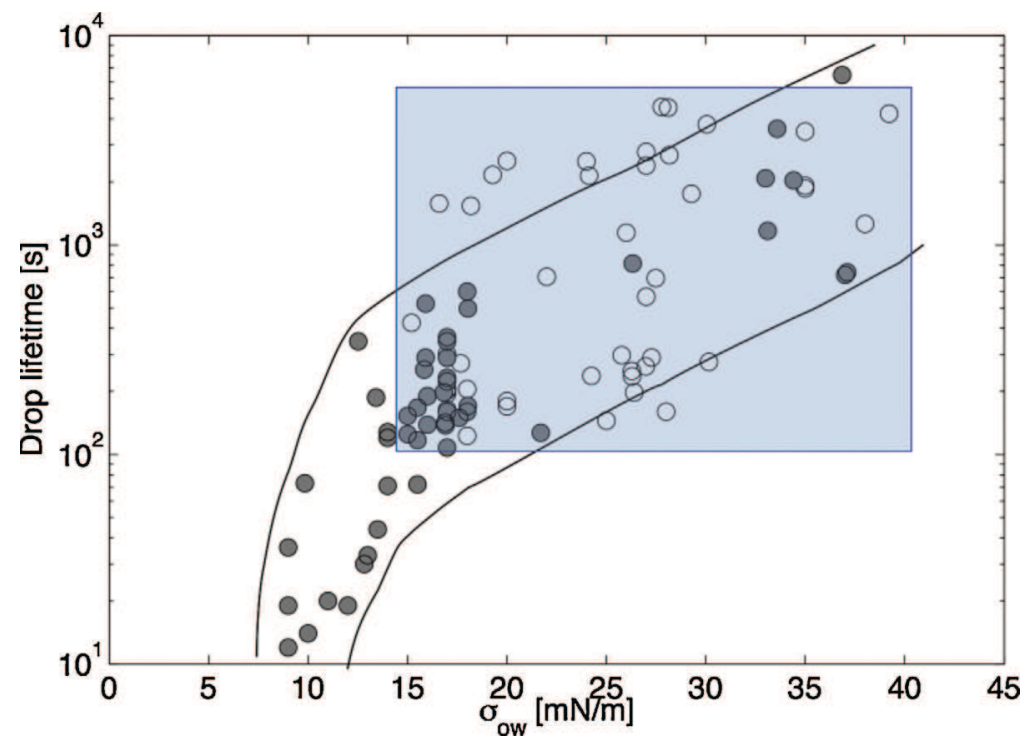

Fig. 11. Drop lifetime versus surface tension for all large drops ( $d>4.4 \mathrm{~mm})$. Empty circles: campaign nP; plain circles: campaign P.

$h_{\mathrm{c}}$ is expected to be of the order of $10 \mathrm{~nm}$. Taking this value for $h_{\mathrm{c}}$, Eq. (14) predicts unrealistically large lifetimes, leading to $T_{\mathrm{L}}=4.1$ $10^{7} \mathrm{~s}$ for $d=4.4 \mathrm{~mm}$. In the same time, the mobile-interface model (corresponding to a contaminant-free interface, Eq. (15)) provides a coalescence time of the order of $1 \mathrm{~s}$, which is unrealistic as well.

Moreover, for a given value of $h_{\mathrm{c}}$, both models predict that $T_{\mathrm{L}}$ should be a decreasing function of $\sigma_{\mathrm{ow}}$, which is contrary to the experimental trend (Fig. 11). The critical thickness is therefore controlled by another mechanism. If we consider that micrometersized particles cause a bridging through the film leading to rapid coalescence, $h_{\mathrm{c}}$ should scale with the particles size. For $d=4.4 \mathrm{~mm}$ Eq. (14) predicts $T_{\mathrm{L}}=4100 \mathrm{~s}$ for $h_{\mathrm{c}}=10^{-6} \mathrm{~m}, T_{\mathrm{L}}=41 \mathrm{~s}$ for $h_{\mathrm{c}}=10^{-5} \mathrm{~m}$, and $T_{\mathrm{L}}=10 \mathrm{~s}$ for $h_{\mathrm{c}}=2.0 \times 10^{-5} \mathrm{~m}$. These values lie in the range of experimental data and in this case, the coalescence time is expected to be a decreasing function of the particle height immersed in the oil film.

Without added particles, dust particles are however probably present at the interface. In some experiments, some isolated large dust particles (order of $10 \mu$ ) could be detected on images. If such large particles are present even randomly, then the presence of 


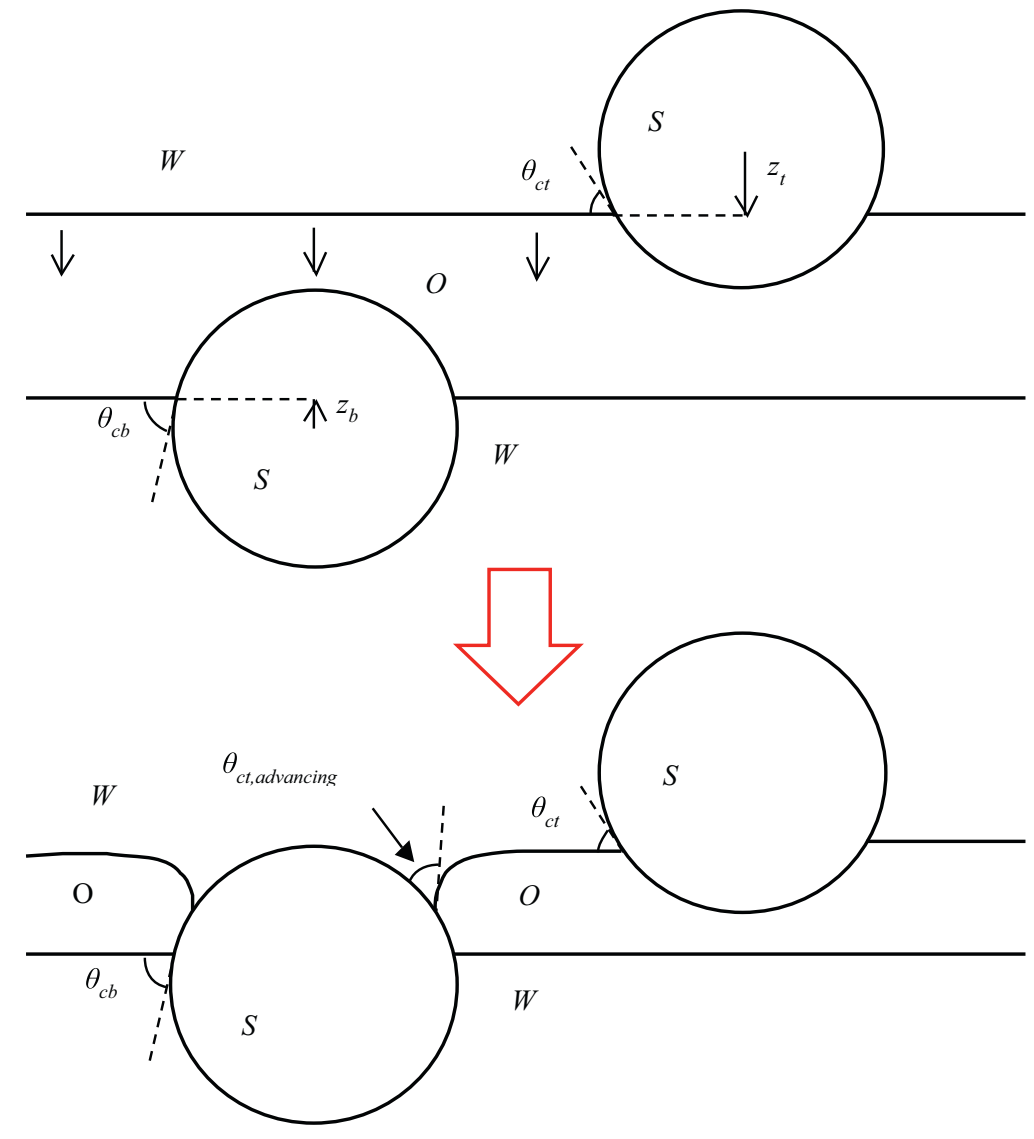

Fig. 12. Illustration of bridging mechanism.

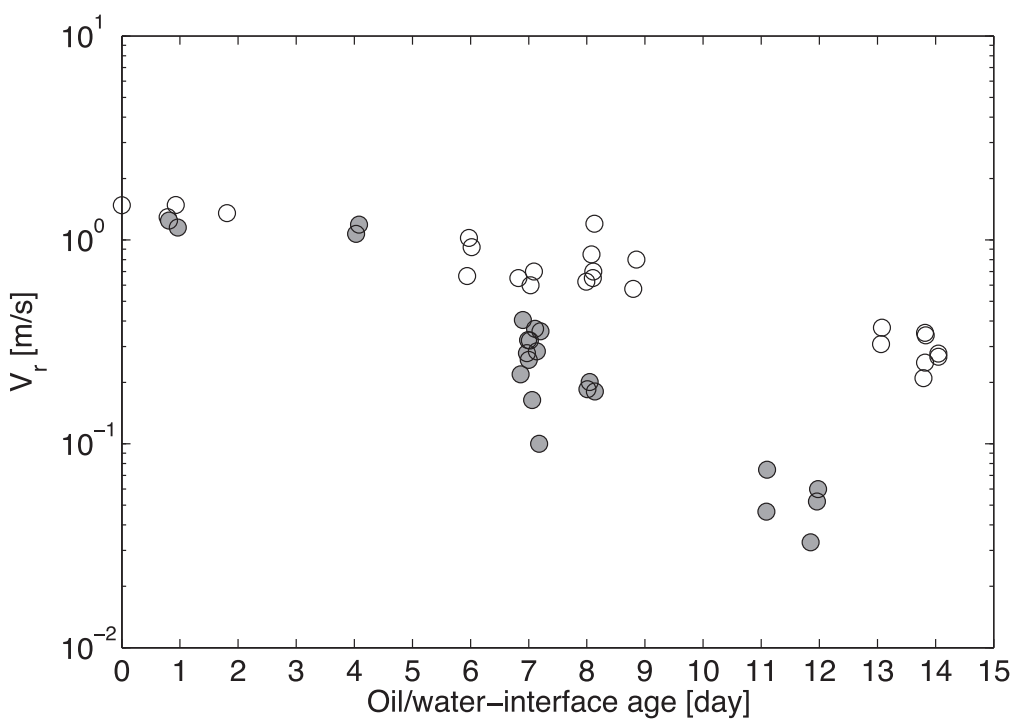

Fig. 13. Evolution of the retraction speed of the oil film with time during campaign $\mathrm{nP}$ (empty circles) and $\mathrm{P}$ (plain circles).

smaller ones is almost certain, but they are not visible since their size is of the order of micron or less, which is less than the resolution of our imaging system $(0.15 \mathrm{px} / \mu \mathrm{m}$ max $)$. The random distribution of lifetimes may thus be related to the random size of dust particles that are present within the oil film during a coalescence event.

When particles with a mean size around $10 \mu \mathrm{m}$ are added, the lifetime is reduced and becomes reproducible provided the physicochemical properties of the interface remain constant. In this case, as predicted by Eq. (14), experiments show that lifetimes increase with the drop diameter (Fig. 10). When the concentration of contaminants adsorbed at the interface changes, not only the interfacial tension is modified but also the average depth of immersion of particles across the interface, and consequently the value of $h_{\mathrm{c}}$.

The bridging mechanism is schematized in Fig. 12 (note that this bridging mechanism is analogue to that described in Dippenar [23] 
or Frye and Berg [25] for particles in foams). In the limit of small Bond numbers, the interface deformation due to the particle weight is small and has been omitted in this figure, so the vertical position of the micro-particle at the water-oil interface is simply determined by the triple line contact angle $\theta_{c}$ in the water phase (thus $\theta_{c}<\pi / 2$ as the particle is hydrophilic). We define as $\theta_{\mathrm{cb}}$ and $\theta_{\mathrm{ct}}$ the contact angles of water with glass particles at the bottom and top interfaces, and $z_{\mathrm{b}}$ and $z_{\mathrm{t}}$ the equilibrium positions that would have the bottom and top interfaces relative to the center of a trapped particle. Taking $\theta_{\mathrm{cb}} \neq \theta_{\mathrm{ct}}$ (or equivalently $\left|z_{\mathrm{b}}\right| \neq\left|z_{\mathrm{t}}\right|$ ) corresponds to the general case (and probably the most common case in real systems) of different wettability between the top and bottom interfaces with particle materials (hence of different interfacial tension). For a particle of radius $r$, we have $z_{\mathrm{b}}=r \cos \theta_{\mathrm{cb}}$ for the bottom interface and $z_{\mathrm{t}}=-r \cos \theta_{\mathrm{ct}}$ for the top interface.

During the drainage process, when the top interface reaches the particle upper surface, the top interface will wet it and try to reach its equilibrium position. As $z_{\mathrm{t}}<z_{\mathrm{b}}$, the equilibrium position of the top interface will be below the bottom interface: the two contact lines will eventually join causing the oil film rupture. The condition $z_{\mathrm{t}}<z_{\mathrm{b}}$ corresponds to $\cos \theta_{\mathrm{cb}}+\cos \theta_{\mathrm{ct}}>0$. Therefore, it is always fulfilled when $\theta_{\mathrm{cb}}<\pi / 2$ and $\theta_{\mathrm{ct}}<\pi / 2$, that is to say when the particle is preferentially wet by water at both interfaces. In the case where $z_{\mathrm{t}}>z_{\mathrm{b}}$, the two contact lines will stay away from each other when bridging occurs, each one anchored to the particle surface at a different height. As opposed to the previous case, this configuration tends to prevent film thinning at the vicinity of the particle, and thus to prevent the occurrence of bridging induced coalescence. This is described in Frye and Berg [25] for particles in foams. Hence small hydrophilic particles are a necessary condition to induce bridging-induced coalescence.

According to this mechanism, the presumed effect of contaminants which is consistent with the correlation between $T_{\mathrm{L}}$ and $\sigma_{\mathrm{ow}}$ (Fig. 11, campaign P), would be to increase in average the height of particles immersed in the oil film phase, leading to a larger critical film thickness, and as a consequence to a shorter lifetime according to Eq. (14). Such an effect therefore corresponds in average to an increase of contact angle of water on the glass particles (but still in a regime of partial wetting below $\pi / 2$ ). The evolution with time of the contact angle of water with $\mu$-particles couldn't be measured. However, there is some qualitative indication of the evolution of the contact angle of water with glass material that can be deduced from Fig. 3. The right image represents a drop resting above an aged interface at day 13 and the undeformed upper part of the drop is clearly visible, indicating that the top of the drop is above the meniscus of the aqueous phase in the cell glass. On the left image, a drop of same diameter is filmed on a freshly formed interface (day 1 ) and its apex is completely below the meniscus. As the interfacial tension has decreased by a factor larger than 2 between the two sequences, the deformation of the interface is stronger at day 13 than at day 1 , so the drop is deeper embedded in the interface on the right image. As a consequence, it can be inferred from these observations that the meniscus made by water on the cell glass walls has decreased, so the contact angle has increased. This picture simply indicates that the cell glass walls are less wetted by water when the interface contamination develops, and even if this cannot be simply extrapolated to the case of the $\mu$-particles, it confirms that it is possible. Such an evolution suggests that in the present case, contaminants adsorb on the particles resting on the oil/water interface for a long time, increasing their hydrophobicity. In order to render model (Eq. (14)) consistent with experiments, such a mechanism also implies that the height of immersion in the oil phase grows faster than $\sigma_{\mathrm{ow}}{ }^{1 / 2}$ as the interface contamination is increasing (i.e. as $\sigma_{\text {ow }}$ is decreasing). Note that a slight increase of contact angle of $10^{\circ}$ from an initial contact angle equal to $30^{\circ}$ leads to an increase

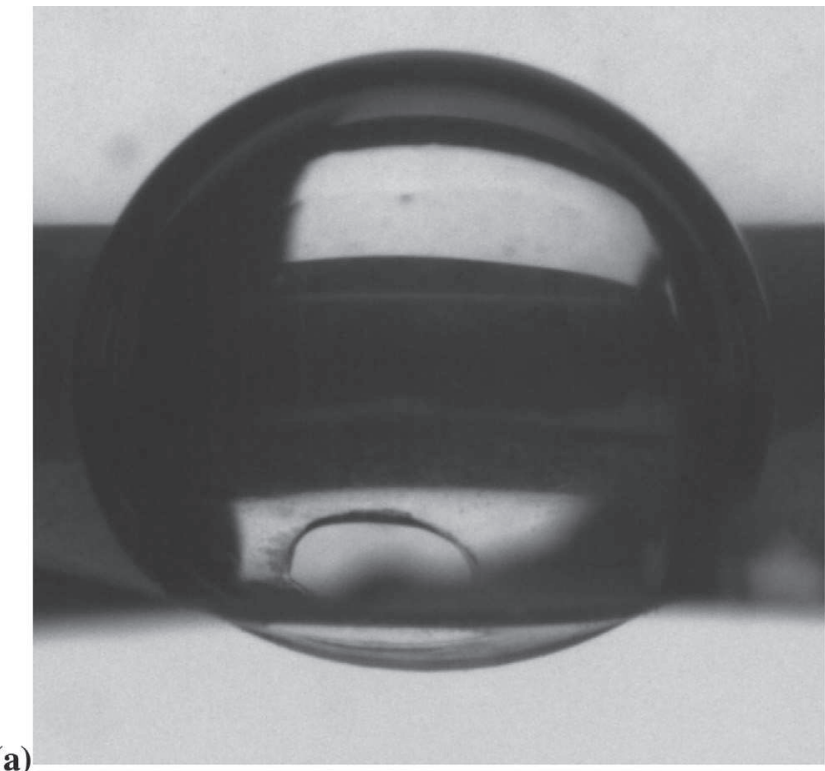

(a)

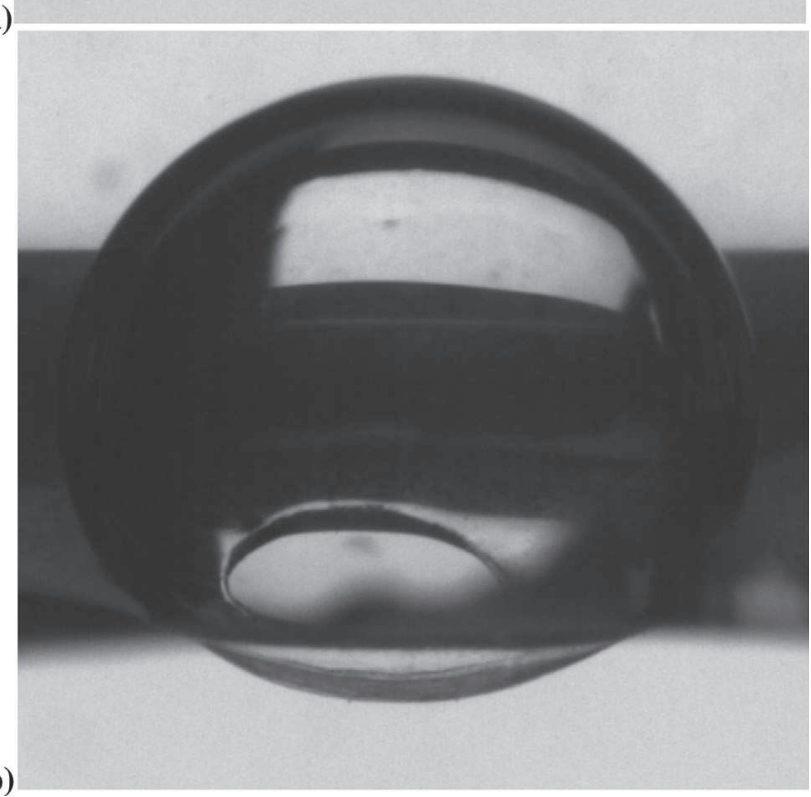

(b)

Fig. 14. Visualization of the oil film bending during film rupture (case of a large drop). The film is observed from above through the drop. Test run performed during last day of campaign nP. Retraction speed was approximately $0.3 \mathrm{~m} \mathrm{~s}^{-1}$.

of the immersed height of the particle in the oil phase by a factor close to 2, leading to a decrease by a factor comprised between 3 and 4 of the drainage time (Eq. (14)). This high sensitivity of the drainage time to the position of floating particles at the interface is another argument in favour of the bridging-induced coalescence mechanism.

\subsection{Film retraction speed}

We consider here the ultimate stage of the coalescence process, which is the retraction of the oil film once it has ruptured. Fig. 13 shows the evolution of the retraction speed $V_{\mathrm{r}}$ with time for campaigns $\mathrm{nP}$ and $\mathrm{P}$. In both cases, it starts from a maximum and then decreases as the oil/water interface ages: from $1.5 \mathrm{~m} \mathrm{~s}^{-1}$ to almost $0.2 \mathrm{~m} \mathrm{~s}^{-1}$ during campaign $\mathrm{nP}$ and from $1.2 \mathrm{~m} \mathrm{~s}^{-1}$ to about $0.03 \mathrm{~m} \mathrm{~s}^{-1}$ during campaign P.

Savva and Bush [35] have calculated the expansion rate of a hole of initial radius $R$ through a planar film of initial thickness $H$, viscosity $\mu$, density $\rho$ and surface tension $\sigma$. The calculation was done 


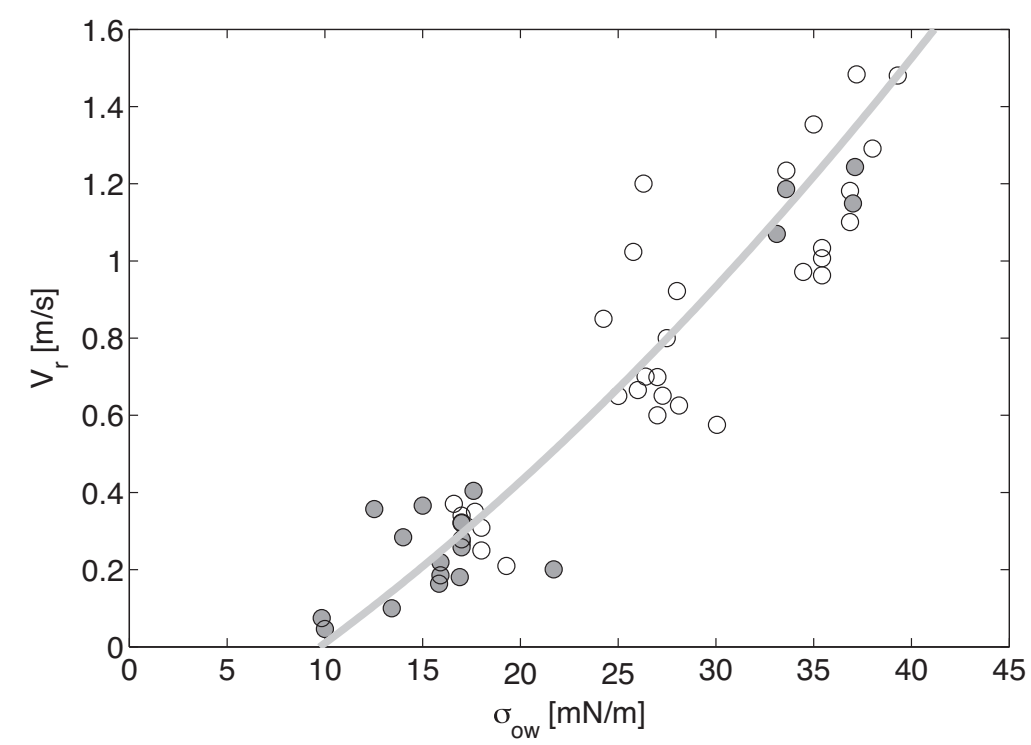

Fig. 15. Retraction speed as a function of oil/water surface tension. Empty circles: campaign nP; plain circles: campaign P.

assuming that the interface remains axisymmetric and symmetric with respect to the middle plane parallel to the sheet. The solution depends on a single dimensionless group:

$G=H /$ ROh, where $\mathrm{Oh}=\mu / \sqrt{2 \rho \mathrm{H} \sigma}$ is the Ohnesorge number that compares viscous to inertial stresses related to interfacial tension. Provided that $R$ is sufficiently large compared to $H$, interfacial tension causes the expansion of the hole. The retraction speed thus results from the balance between interfacial tension that drives the expansion and viscous forces and inertia that oppose to it. Starting from zero at the onset of hole formation, it then increases with time until it reaches a constant value, which is independent of viscosity, known as the Taylor-Culik speed, $u_{c}=\sqrt{2 \sigma / \rho H}$. Since our measurements are performed during the stage where the hole expands over an almost flat portion of the film, this model should be relevant to analyze the present situation. In particular, due to the fact that the retraction speed remains nearly constant during hole expansion, the film thickness $h_{\mathrm{c}}=\mathrm{H}$ can be estimated assuming $V_{\mathrm{r}}=\mathrm{u}_{\mathrm{c}}$. Taking $\mathrm{V}_{\mathrm{r}}=1.4 \mathrm{~m} \mathrm{~s}^{-1}$ and $\sigma_{\mathrm{ow}}=40 \mathrm{mN} \mathrm{m}^{-1}$ as typical values for the beginning of a campaign, it yields $H=42 \mu \mathrm{m}$, which is significantly larger than expected values for $h_{\mathrm{c}}$. Taking $V_{\mathrm{r}}=0.2 \mathrm{~m} \mathrm{~s}^{-1}$ and $\sigma_{\mathrm{ow}}=17 \mathrm{mN} \mathrm{m}^{-1}$ as typical values for the end of campaign $\mathrm{nP}$, it yields $H=2 \mathrm{~mm}$, which is unrealistic. One can argue that the retraction speed has not yet reached its maximal value, and that the Taylor-Culik expression therefore overestimates the retraction speed. But in that case, according to the theory of Savva \& Bush, the film retraction should accelerate, which is not verified by experimental observations. Moreover, whatever the value of group G, the theory of Savva \& Bush [35] predicts that the retraction speed should be a decreasing function of the film thickness. However, especially during campaign $\mathrm{nP}$, there are several examples of successive test runs with similar surface tension and drop diameter but with contrasted coalescence times, and yet yielding similar retraction speeds. Also, in Fig. 13, it can be observed that during the first 3 days, adding particles hasn't changed significantly the film retraction speed. If the coalescence time is related to the film thickness, then it seems surprising that the retraction speed be not correlated to this quantity.

The discrepancy between the measured retraction speeds and the theory of Savva and Bush can be understood through a more careful examination of the hole rim during its expansion. Fig. 14 shows that the oil film is indeed bent towards inside of the drop. Such a phenomenon is expected if we consider that interfacial ten- sions of the upper and lower sides of the oil film are different. As a new drop is produced for each test, the oil/drop interface is always fresh and its interfacial tension, noted $\sigma_{\text {od }}$, is nearly constant, close to $35 \mathrm{mN} \mathrm{m}^{-1}$. In return, oil/water interface contamination is growing day after day and its interfacial tension $\sigma_{\text {ow }}$, keeps on decreasing with time. After the onset of film rupture, interfacial tension hence pulls stronger the upper than the lower face of the film, which tends to bend upward. As a result, an important drag force will be exerted on the film tip, slowing down the film retraction acceleration. This mechanism offers a plausible explanation of the low values of the retraction velocities compared to those predicted for a plane axisymmetric film. It also implies that the retraction speed has to be a decreasing function of the difference $\sigma_{\mathrm{od}}-\sigma_{\mathrm{ow}}$, and therefore an increasing function of $\sigma_{\text {ow }}$.

In Fig. $15, V_{\mathrm{r}}$ is plotted against $\sigma_{\mathrm{ow}}$ for all tests of both campaigns. Although the dispersion is important (partly due to the weak accuracy on the retraction speed measurement), experimental data regroup on an increasing master curve (except for a few tests belonging to the $\mathrm{nP}$ campaign for $\sigma_{\text {ow }}$ values lying in the range between 25 and $30 \mathrm{mN} \mathrm{m}^{-1}$ ). The increase of $V_{\mathrm{r}}$ is much stronger than the $\sigma_{\mathrm{ow}}{ }^{1 / 2}$ evolution predicted by the Taylor-Culik expression, which only accounts for the increase of the driving force when interfacial tension becomes greater. We can therefore conclude that in these experiments, the retraction speed is not directly correlated to the film thickness but mainly controlled by the surface tension difference between both sides of the film.

A possible way to match the oil/drop and the oil/water interfacial tensions and tend towards the planar geometry of the film during retraction is to add surfactants at a well-controlled concentration. However, prior to this experiment, it seems relevant to test the sensitivity of the film bending effect to the interfacial tension difference, a study that can be achieved by means of numerical simulations. This work is currently under development.

\section{Conclusion}

Following the argument of de Gennes [21], the present work focuses on the role of micro-particles trapped at the interface on coalescence process dynamics of contaminated interfaces.

We have investigated in this work the influence of $\mu$-particles at an oil-water interface on the coalescence of millimetre size settling water drops. Analysis of drop settling velocity first indicates that 
the oil/drop interface is immobilized in all cases by the presence of adsorbed surface-active species at the interface. As the coalescence experiments go on, these contaminants accumulate with time at the oil/water interface.

When no solid particle is added, measured drop lifetimes are randomly ranging between a few tens and several thousand seconds and no correlation between the drop lifetime and the drop size or interfacial tension of the oil/water interface is observed. When $10-\mu \mathrm{m}$ glass particles are trapped at the oil/water interface, drop lifetimes decrease and data scattering is reduced. Over periods from one to three hours during which the physicochemical properties of the oil/water interface do not evolve, lifetimes are observed to be reproducible and to increase with drop diameters - as expected from classical models of interface deformation and film drainage [33]. Moreover, over longer periods of time during which the oil/water interface contamination is increasing, drop lifetime is clearly correlated with the oil/water interfacial tension: the lower the interfacial tension, the shorter the drop lifetime, a result which is not supported by classical drainage models (with mobile or immobile interfaces). Introducing large glass particles of a given size can therefore make the coalescence process reproducible. This suggests that the drainage process is in general (i.e. for non ultra-pure systems) reproducible but not the critical thickness $h_{\mathrm{c}}$ at which the film ruptures. In the presence of $10-\mu \mathrm{m}$ glass particles, $h_{\mathrm{c}}$ is likely to be scaled by the average height of particle immersion in the oil film, which depends directly on the oil/water/particle contact angle, and which is larger than the critical thicknesses related to any random causes of film rupture. The contact angle, and thus $h_{\mathrm{c}}$, depends of the interface contamination: this means that contamination has an indirect influence on the drop rest times by modifying $h_{\mathrm{c}}$. Through bridging mechanism, the random presence of solid contaminants is therefore a probable major cause of the stochastic character of coalescence process in many practical situations.

Unlike the case of a retracting axisymmetric and planar film, the retraction speed is not controlled here by the film thickness $h_{\mathrm{c}}$ after it has ruptured (Taylor-Culik speed). Because the interfacial tension of the drop (which has a fresh interface) is larger than that of the oil/water interface (which is ageing day after day), the oil film bends upwards along the hole rim. The bended shape increases the fluid resistance to the hole expansion and decreases the retraction velocity. The lower the oil/water interfacial tension, the larger the interfacial tension difference between the upper and the lower part of the film, the larger the bending, and the lower the retraction speed. This mechanism explains the nice collapse of the measured retraction speeds around a master growing function of $\sigma_{\mathrm{ow}}$.

The present conclusions about the coalescence time and the retraction speed provide new insights that are relevant for the understanding and the modeling of the coalescence process in practical situations where interfaces are contaminated by surfaceactive molecules and micro-particles.

\section{Acknowledgements}

Authors are grateful to FACE center in Norway and Fédération de Recherche FERMaT of Toulouse for financial support.

\section{References}

[1] S.L. Kokal, Crude oil emulsions, in: J.R. Fanchi (Ed.), Petroleum Engineering Handbook, Vol. I, General Engineering, Society of Petroleum Engineers, Richardson, Texas, 2006, pp. 533-570, ISBN 978-1-55563-113-0.
[2] E.G. Cockbain, T.S. McRoberts, The stability of elementary emulsion drops and emulsions, J. Colloid Sci. 8 (1953) 440-451.

[3] T. Gillespie, E.K. Rideal, The coalescence of drops at an oil-water interface Trans, Faraday Soc. 52 (1956) 173-183.

[4] G.A.H. Elton, R.G. Picknett, The coalescence of aqueous droplets with an oil/water interface, Butterworths, London, in: Proceedings of the 2nd International Congress of Surface Activity, vol. 1, 1957, p. 288.

[5] L.E. Nielsen, R. Wall, G. Adams, The coalescence of liquid drops at oil-water interfaces, J. Colloid Sci. 13 (1958) 441-458.

[6] G.E. Charles, S.G. Mason, The coalescence of drop with flat liquid/liquid interfaces, J. Colloid Sci. 15 (3) (1960) 236-267.

[7] S. Hartland, The coalescence of a liquid drop at a iquid?liquid interface. Part I: drop shape, Trans. Institution Chem. Eng. 45 (1967) 97-101.

[9] S. Hartland, The coalescence of a liquid drop at a liquid-liquid interface. Part III: film rupture, Trans. Institution Chem. Eng. 45 (1967) 109-114.

[10] S. Hartland, The coalescence of a liquid drop at a liquid-liquid interface. Part V: the effect of surface active agents, Trans. Institut. Chem. Eng. 46 (1968) 275-282.

[11] T.D. Hodgson, J.C. Lee, The effect of surfactants on the coalescence of a drop at an interface I, J. Colloid Interface Sci. 30 (1) (1969) 94-108.

[12] A.F. Jones, S.D.R. Wilson, The film drainage problem in droplet coalescence, J. Fluid Mech. 87 (2) (1978) 263-288.

13] E.S. Basheva, T.D. Gurkov, I.B. Ivanov, G.B. Bantchev, B. Campbell, R.P. Borwanker, Size dependence of the stability of emulsion drops pressed against a large interface, Langmuir 15 (60) (1999) 6764-6769.

[14] T.D. Hodgson, D.R. Woods, The effect of surfactants on the coalescence of a drop at an interface II, J. Colloid Interface Sci. 30 (4) (1969) 429-446.

[15] S.B. Lang, C.R. Wilke, A hydrodynamic mechanism for the coalescence of liquid drops. I. Theory of coalescence at a planar interface, Ind. Eng. Chem. Fundam. 10 (3) (1971) 329-340.

[16] S.B. Lang, C.R. Wilke, A hydrodynamic mechanism for the coalescence of liquid drops. II. Experimental studies, Ind. Eng. Chem. Fundam. 10 (3) (1971) 341-352.

[17] D.R. Woods, K.A. Burrill, The stability of emulsions, J. Electroanal. Chem. 37 (1972) 191-213.

18] Z. Mohamed-Kassim, E.K. Longmire, Drop coalescence through a liquid/liquid interface, Phys. Fluids 16 (7) (2004) 2170-2181.

[19] S. Chen, An experimental study of the coalescence between a drop and an interface in Newtonian and polymeric liquids, Phys. Fluids 18 (92103) (2006) $1-14$.

[20] G. Bozzano, M. Dente, Modelling the drop coalescence at the interface of two liquids, Comput. Chem. Eng. 35 (5) (2011) 901-906.

[21] P.G. de Gennes, Some remarks on coalescence in emulsions of foams, Chem. Eng. Sci. 56 (2001) 5449-5450.

[22] P.R. Garrett, The effect of polytetrafluoroethylene particles on the foamability of aqueous surfactant solutions, J. Colloid Interface Sci. 69 (1) (1979) 107-121.

[23] A. Dippenaar, The destabilization of froth by solids. I. The mechanism of film rupture, Int. J. Miner. Process. 9 (1982) 1-14.

[24] A. Dippenaar, The destabilization of froth by solids. II. The rate-determining step, Int. J. Miner. Process. 9 (1982) 15-27.

[25] G.C. Frye, J.C. Berg, Antifoam action by solid particles, J. Colloid Interface Sci. 127 (1) (1989) 222-238.

[26] D. Vella, D.G. Lee, H.Y. Kim, The load supported by small floating objects, Langmuir 22 (2006) 5979-5981.

[27] E. Klaseboer, E.J.P. Chevaillier, C. Gourdon, O. Masbernat, Film drainage between colliding drops at constant approach velocity: experiments and modelling, J. Colloid Interface Sci. 229 (2000) 274-285.

[28] A. Miyamura, S. Iwasaki, T. Ishii, Experimental wall correction factors of single solid spheres in triangular and square cylinders, and parallel plates, Int. J. Multiphase Flow 7 (1981) 41-46.

[29] R. Satapathy, W. Smith, The motion of single immiscible drops through a liquid, J. Fluid Mech. 10 (4) (1961) 561-570.

[30] T.D. Taylor, A. Acrivos, On the deformation and drag of a falling viscous drop at low Reynolds number, J. Fluid Mech. 18 (3) (1964) 466-476.

[31] K.D. Danov, T.D. Gurkov, T. Dimitrova, I.B. Ivanov, D. Smith, Hydrodynamic theory for spontaneously growing dimple in emulsion films with surfactant mass transfer, J. Colloid Interface Sci. 188 (1997) 313-324.

[32] J.P. Chevaillier, E. Klaseboer, O. Masbernat, C. Gourdon, Effect of mass transfer on the film drainage between colliding drops, J. Colloid Interface Sci. 299 (2006) 472-485.

[33] A.K. Chesters, The modelling of coalescence processes in fluid-liquid dispersions: a review of current understanding, Chem. Eng. Res. Des. 69 (A4) (1991) 259-270.

[34] S.G. Yiantsios, R.H. Davis, On the buoyancy-driven motion of a drop towards a rigid surface or a deformable interface, J. Fluid Mech. 217 (1990) 547-573.

[35] N. Savva, N.J.W.M. Bush, Viscous sheet retraction, J. Fluid Mech. 626 (2009) 211-240. 